\title{
Spin dynamics investigations for the electric dipole moment experiment
}

\author{
F. Rathmann $\oplus^{1,{ }^{*}}$ N. N. Nikolaev $\oplus^{2,3}$ and J. Slim $\oplus^{4}$ \\ ${ }^{1}$ Institut für Kernphysik, Forschungszentrum Jülich, 52425 Jülich, Germany \\ ${ }^{2}$ L.D. Landau Institute for Theoretical Physics, 142432 Chernogolovka, Russia \\ ${ }^{3}$ Moscow Institute for Physics and Technology, 141700 Dolgoprudny, Russia \\ ${ }^{4}$ III. Physikalisches Institut B, RWTH Aachen University, 52056 Aachen, Germany
}

(Received 11 November 2019; accepted 29 January 2020; published 27 February 2020)

\begin{abstract}
Precision experiments, such as the search for a deuteron electric dipole moment using storage rings like COSY, demand for an understanding of the spin dynamics with unprecedented accuracy. In such an enterprise, numerical predictions play a crucial role for the development and later application of spintracking algorithms. Various measurement concepts involving polarization effects induced by an rf Wien filter and static solenoids in COSY are discussed. The matrix formalism, applied here, deals solely with spin rotations on the closed orbit of the machine, and is intended to provide numerical guidance for the development of beam and spin-tracking codes for rings that employ realistic descriptions of the electric and magnetic bending and focusing elements, solenoids, etc., and a realistically modeled rf Wien filter.
\end{abstract}

DOI: 10.1103/PhysRevAccelBeams.23.024601

\section{INTRODUCTION}

The Standard Model (SM) of particle physics is not capable to account for the apparent matter-antimatter asymmetry of the Universe. Physics beyond the SM is required and it is either probed by employing high energies (e.g., at LHC), or by striving for ultimate precision and sensitivity (e.g., in the search for electric dipole moments). Permanent electric dipole moments (EDMs) of particles violate both time-reversal $(\mathcal{T})$ and parity $(\mathcal{P})$ invariance, and are via the $\mathcal{C P} \mathcal{T}$ theorem also $\mathcal{C P}$ violating. Finding an EDM would be a strong indication for physics beyond the SM, and pushing upper limits further provides crucial tests for any corresponding theoretical model, e.g., SUSY.

Up to now, EDM searches mostly focused on neutral systems (neutrons, atoms, and molecules). Storage rings, however, offer the possibility to measure EDMs of charged particles by observing the influence of the $\mathrm{EDM}$ on the spin motion in the ring. These direct searches of e.g., proton and deuteron EDMs bear the potential to reach sensitivities beyond $10^{-29} \mathrm{e} \mathrm{cm}$. Since the cooler synchrotron $\operatorname{COSY}^{1}$ at the Forschungszentrum Jülich

\footnotetext{
*f.rathmann@fz-juelich.de

${ }^{1}$ The synchrotron and storage ring COSY accelerates and stores unpolarized and polarized proton or deuteron beams in the momentum range of 0.3 to $3.65 \mathrm{GeV} / c$ [1].

Published by the American Physical Society under the terms of the Creative Commons Attribution 4.0 International license. Further distribution of this work must maintain attribution to the author(s) and the published article's title, journal citation, and DOI.
}

provides polarized protons and deuterons up to momenta of $3.7 \mathrm{GeV} / c$, it constitutes an ideal testing ground and a starting point for such an experimental program.

The investigations presented here, carried out in the framework of the JEDI collaboration, ${ }^{2}$ are relevant for the preparation of the deuteron EDM measurement [3]. A radio-frequency (rf) Wien filter (WF) [4-6] makes it possible to carry out EDM measurements in a conventional magnetic machine like COSY. The idea is to look for an EDM-driven resonant rotation of the deuteron spins from the horizontal to the vertical direction and vice versa, generated by the rf Wien filter at the spin precession frequency $[7,8]$.

The search for EDMs of protons, deuterons, and heavier nuclei using storage rings $[2,9,10]$ is part of an extensive world-wide effort to push further the frontiers of precision spin dynamics of polarized particles in storage rings. In this context, the JEDI results prompted the formation of the new Charged Particle Electric Dipole Moment (CPEDM) collaboration, ${ }^{3}$ which aims at the development of a purely electric prototype storage ring, with drastically enhanced sensitivities to the EDM of protons and deuterons, compared to what is presently feasible at COSY $[3,11]$.

Precision experiments, such as the EDM searches, demand for an understanding of the spin dynamics with unprecedented accuracy, keeping in mind that the ultimate

\footnotetext{
${ }^{2}$ The proposals of the JEDI collaboration (Jülich Electric Dipole moment Investigations) are available from [2].

${ }^{3}$ The website of the Charged Particle Electric Dipole Moment Collaboration is available via http://pbc. web.cern.ch/edm/edmdefault.htm.
} 
aim is to measure EDMs with a sensitivity up to 15 orders in magnitude better than the magnetic dipole moment (MDM) of the stored particles.

The description of the physics of the applied approach, called $r f$ Wien filter mapping, is discussed further in a forthcoming separate publication. The theoretical understanding of the method and its experimental exploitation are prerequisites for the planned EDM experiments at COSY [2], and will also have an impact on the design of future dedicated EDM storage rings [11].

This paper discusses various polarization effects that are induced by the rf Wien filter and static solenoids in the ring. The approach taken here strongly simplifies the machine lattice, and deals solely with spin rotations on the closed orbit $[12,13]$, described by the $\mathbf{S O}(\mathbf{3})$ formalism. One aim of the work is to obtain a basic understanding about the interplay of spin rotations in a magnetic ring equipped with an rf Wien filter and solenoid magnets, under the simplifying assumption mentioned above. In an ideal machine with perfect alignment of the magnetic elements, the spin rotations on the closed orbit are generated primarily by the dipole magnets, therefore, for the time being, spin rotations in the quadrupole magnets are not considered.

As we shall demonstrate below, even with an idealized ring, the parametric $\mathrm{rf}$ resonance-driven spin rotations reveal quite a reach pattern of spin dynamics. Our results set the background for more realistic spin tracking calculations, based on recent geodetic surveys of COSY that make available position offsets, roll, and inclination parameters for the quadrupole and dipole magnets. The treatment of the spin transport through these individually misaligned magnetic elements, can, however, be readily incorporated in the applied matrix formalism. Besides that, the spin dynamics simulations carried out in the framework of the present paper will serve as a valuable cross-check of the analytic approximate treatment of the parametric spin resonance, based on the Bogolyubov-Krylov-Mitropolsky averaging technique [14].

The JEDI collaboration is presently implementing a beam-based alignment scheme at COSY, which aims at providing optimized beam-transfer properties of the quadrupole and dipole magnets in the ring, intending to make the beam orbit as planar as possible [15]. Once this is accomplished, the spin dynamics in the ring will be largely governed by the misaligned dipoles alone. Thus effectively, the approach described here will appropriately describe an EDM experiment using an rf Wien filter in a beam-based aligned ring.

The paper is organized as follows. In Sec. II, the effect of an EDM on the spin evolution in a ring is discussed in terms of the Thomas-BMT equation [12]. The inclusion of an $\mathrm{rf}$ Wien filter in an otherwise ideal ring is treated in Sec. III, while the polarization evolution with an $\mathrm{rf}$ Wien filter and additional solenoids is discussed in Sec. IV. The main findings are summarized in the conclusions in Sec. V. A brief outlook into additional aspects planned to be investigated using the simulation approach taken here in the near future is also given.

\section{SPIN ROTATIONS IN THE RING}

\section{A. Thomas-BMT equation}

Below, the basic formalism to describe the spin evolution in electric and magnetic fields is briefly reiterated. The generalized form of the Thomas-BMT equation describes the spin motion of a particle with spin $\vec{S}$ in an arbitrary electric $(\vec{E})$ and magnetic field $(\vec{B})$. Including EDMs (in SI units), it reads [16]

$$
\frac{\mathrm{d} \vec{S}}{\mathrm{~d} t}=\underbrace{\left(\vec{\Omega}^{\mathrm{MDM}}+\vec{\Omega}^{\mathrm{EDM}}\right)}_{=\vec{\Omega}^{\mathrm{tot}}} \times \vec{S},
$$

where

$$
\begin{aligned}
\vec{\Omega}^{\mathrm{MDM}}= & -\frac{q}{m}\left[\left(G+\frac{1}{\gamma}\right) \vec{B}-\frac{G \gamma}{\gamma+1}(\vec{\beta} \cdot \vec{B}) \vec{\beta}\right. \\
& \left.-\left(G+\frac{1}{\gamma+1}\right) \frac{\vec{\beta} \times \vec{E}}{c}\right], \\
\vec{\Omega}^{\mathrm{EDM}}= & -\frac{q}{m c} \frac{\eta_{\mathrm{EDM}}}{2}\left[\vec{E}-\frac{\gamma}{\gamma+1}(\vec{\beta} \cdot \vec{E}) \vec{\beta}+c \vec{\beta} \times \vec{B}\right] .
\end{aligned}
$$

Here $m, \gamma$, and $\vec{\beta}$ are the mass, Lorentz factor, and the velocity of a particle in units of the speed of light $c$ in vacuum. $\vec{S}$ (in units of $\hbar$ ) is given in the particle rest frame, and the fields $\vec{E}$ and $\vec{B}$ are in the laboratory system. The magnetic dipole moment $\vec{\mu}$ (MDM) and the electric dipole moment $\vec{d}$ (EDM) are defined via the dimensionless Landéfactor $g$ and $\eta_{\mathrm{EDM}}$

$$
\vec{\mu}=g \frac{q}{2 m} \vec{S}, \quad \text { and } \quad \vec{d}=\eta_{\mathrm{EDM}} \frac{q}{2 m c} \vec{S} .
$$

The magnetic anomaly is given by

$$
G=\frac{g-2}{2} .
$$

\section{B. EDM tilt angle $\boldsymbol{\xi}$ from the Thomas-BMT equation}

In an ideal machine without unwanted magnetic fields, the axis about which the particle spins precess is given by the purely vertical magnetic field $\vec{B}=\vec{B}_{\perp}=B_{\perp} \cdot \vec{e}_{y}$. Equating the COSY orbital angular velocity $\Omega_{\text {rev }}=2 \pi f_{\text {rev }}$ and the relativistic cyclotron angular velocity

$$
\vec{\Omega}_{\mathrm{rev}}=\left(\begin{array}{c}
0 \\
2 \pi \cdot f_{\mathrm{rev}} \\
0
\end{array}\right)=\vec{\Omega}_{\mathrm{cyc}}=-\frac{q}{\gamma m}\left(B_{\perp}-\frac{\vec{\beta} \times \vec{E}}{\beta^{2} c}\right),
$$

yields, for $\vec{E}=0$ with the parameters given in Table I, a vertical magnetic field of 
TABLE I. Parameters of the deuteron kinematics, the COSY ring, the deuteron elementary quantities, the electric dipole moment (EDM) assumed, and the field integrals of the idealized $\mathrm{rf}$ Wien filter (to eight decimal places). The deuteron momentum $P$ is used to specify the deuteron kinetic energy $T$, and the Lorentz factors $\beta$ and $\gamma$. The COSY circumference $\ell_{\operatorname{COSY}}$ is used to specify the COSY revolution frequency $f_{\text {rev }}$ and the spin-precession frequency $f_{s}$. The deuteron mass $m$ and the deuteron $g$ factor, taken from the NIST database [17] (not from the most recent one), are used to specify $G$. The deuteron EDM $d$ is used to quantify $\eta_{\mathrm{EDM}}$ and $\xi_{\mathrm{EDM}}$.

\begin{tabular}{llc}
\hline \hline Quantity & & Value \\
\hline Deuteron momentum (lab) & $P$ & $970.00000000 \mathrm{MeV} / c$ \\
Deuteron energy (lab) & $T$ & $235.97981668 \mathrm{MeV}$ \\
Lorentz factor (lab) & $\gamma$ & 0.45936891 \\
Lorentz factor (lab) & $\ell_{\mathrm{COSY}}$ & 1.12581478 \\
COSY circumference & $f_{\text {rev }}$ & $183.57200000 \mathrm{~m}$ \\
COSY revolution frequency & $f_{s}$ & $750197.93487176 \mathrm{~Hz}$ \\
COSY spin precession frequency & $m$ & $120764.75147311 \mathrm{~Hz}$ \\
Deuteron mass & $g$ & $1875.61279300 \mathrm{MeV}$ \\
Deuteron $g$ factor & $G$ & 1.71402546 \\
Deuteron $G=(g-2) / 2$ & $d$ & -0.14298727 \\
Deuteron EDM & $\eta_{\mathrm{EDM}}$ & $10^{-20} e \mathrm{~cm}$ \\
Deuteron dimensionless $\eta_{\mathrm{EDM}}$ & $\xi_{\mathrm{EDM}}$ & $1.90102028 \times 10^{-6}$ \\
Deuteron EDM tilt angle & $f_{\mathrm{ampl}}$ & $-3.05366207 \times 10^{-6}$ \\
rf Wien filter field amplification factor & $\int E_{x}^{\mathrm{WF}} \mathrm{d} z$ & $10^{3}$ \\
rf Wien filter electric field integral & $\int B_{y}^{\mathrm{WF}} \mathrm{d} z$ & $2.20000000 \times 10^{6} \mathrm{~V}$ \\
rf Wien filter magnetic field integral & $\ell_{\mathrm{WF}}$ & $1.59749820 \times 10^{-2} \mathrm{~T} \mathrm{~m}$ \\
rf Wien filter length & & $1.55000000 \mathrm{~m}$ \\
\hline \hline
\end{tabular}

$$
\vec{B}_{\perp}=\left(\begin{array}{c}
0 \\
1.1075 \times 10^{-1} \\
0
\end{array}\right) \mathrm{T} .
$$

This value can be considered as the magnetic field that corresponds to an equivalent COSY ring where the magnetic fields are evenly distributed.

Inserting $\vec{B}$ from Eq. (6) and $\vec{E}=0$ into Eq. (2), yields for the angular velocity in the particle rest system

$$
\begin{aligned}
\vec{\Omega}^{\mathrm{tot}} & =\vec{\Omega}^{\mathrm{MDM}}+\vec{\Omega}^{\mathrm{EDM}}=-\frac{q}{m}\left(\begin{array}{c}
\frac{1}{2} \eta_{\mathrm{EDM}} \beta \\
G+\frac{1}{\gamma} \\
0
\end{array}\right) B_{\perp} \\
& =\left(\begin{array}{c}
-2.3171 \\
3954845.3298 \\
0.0000
\end{array}\right) \mathrm{s}^{-1} .
\end{aligned}
$$

In the laboratory system, however, we observe with the parameters of Table I the angular velocity with respect to the cyclotron motion of the momentum,

$$
\begin{aligned}
\vec{\Omega}^{\mathrm{Lab}} & =\vec{\Omega}^{\mathrm{tot}}-\vec{\Omega}_{\mathrm{rev}}=-\frac{q}{m}\left(\begin{array}{c}
\frac{1}{2} \eta_{\mathrm{EDM}} \beta \\
G \\
0
\end{array}\right) B_{\perp} \\
& =\left(\begin{array}{c}
-2.3171 \\
-758787.3121 \\
0.0000
\end{array}\right) \mathrm{s}^{-1}
\end{aligned}
$$

where $\vec{\Omega}_{\text {rev }}$ denotes the COSY angular velocity along $\vec{e}_{y}$. The spin-precession frequency yields the familiar value of

$$
f_{s}=\frac{\vec{\Omega}^{\mathrm{Lab}}}{2 \pi}=\left(\begin{array}{c}
-0.3688 \\
-120764.7515 \\
0.0000
\end{array}\right) \mathrm{s}^{-1},
$$

which is also listed in Table I. The angle by which the stable spin axis is tilted, i.e., the angle between $\vec{\Omega}^{\mathrm{Lab}}$ and $\vec{e}_{y}$ is obtained by evaluating

$$
\xi=\arctan \left|\frac{\vec{\Omega}^{\mathrm{Lab}} \times \vec{e}_{y}}{\vec{\Omega}^{\mathrm{Lab}} \cdot \vec{e}_{y}}\right| .
$$

Inspecting Eq. (8), the effect of an EDM in a magnetic machine can be expressed by the tilt of the stable spin axis away from the vertical orientation in the ring, given by ${ }^{4}$

$$
\tan \xi_{\mathrm{EDM}}=\frac{\eta_{\mathrm{EDM}} \beta}{2 G}
$$

For an assumed EDM of $d=1 \times 10^{-20} e \mathrm{~cm}$, and for deuterons at a momentum of $970 \mathrm{MeV} / c$, Eqs. (3) and (11) yield $\eta_{\mathrm{EDM}}$ and $\xi_{\mathrm{EDM}}$, as listed in Table I.

\footnotetext{
${ }^{4}$ In Eq. (11), an additional factor of 2 has been inserted in the denominator, correcting Eq. (10) of [18].
} 


\section{Rotation matrices}

Our description of the spin dynamics is based on the $\mathbf{S O}(3)$ formalism. A rotation by an angle $\theta$ around an arbitrary axis given by the unit vector $\vec{n}=\left(n_{1}, n_{2}, n_{3}\right)$ is described by the matrix [19]

$$
\mathbf{R}(\vec{n}, \theta)=\left(\begin{array}{lll}
b_{11} & b_{12} & b_{13} \\
b_{21} & b_{22} & b_{23} \\
b_{31} & b_{32} & b_{33}
\end{array}\right),
$$

with

$$
\begin{aligned}
& b_{11}=\cos \theta+n_{1}^{2}(1-\cos \theta) \\
& b_{12}=n_{1} n_{2}(1-\cos \theta)-n_{3} \sin \theta \\
& b_{13}=n_{1} n_{3}(1-\cos \theta)+n_{2} \sin \theta \\
& b_{21}=n_{1} n_{2}(1-\cos \theta)+n_{3} \sin \theta \\
& b_{22}=\cos \theta+n_{2}^{2}(1-\cos \theta) \\
& b_{23}=n_{2} n_{3}(1-\cos \theta)-n_{1} \sin \theta \\
& b_{31}=n_{1} n_{3}(1-\cos \theta)-n_{2} \sin \theta \\
& b_{32}=n_{2} n_{3}(1-\cos \theta)+n_{1} \sin \theta \\
& b_{33}=\cos \theta+n_{3}^{2}(1-\cos \theta) .
\end{aligned}
$$

\section{One turn spin rotation matrix with EDM}

With a nonvanishing EDM, in the rotation matrix of Eq. (12), the spins do not precess anymore around the vertical axis $\vec{e}_{y}$, but rather around the direction given by

$$
\vec{c}\left(\xi_{\mathrm{EDM}}\right)=\left(\begin{array}{l}
c_{1} \\
c_{2} \\
c_{3}
\end{array}\right)=\left(\begin{array}{c}
\sin \xi_{\mathrm{EDM}} \\
\cos \xi_{\mathrm{EDM}} \\
0
\end{array}\right) .
$$

Therefore, the ring rotation matrix can be obtained by inserting into Eq. (12) the coefficients $c_{1}, c_{2}, c_{3}$ from Eq. (14), and by setting

$$
\theta:=\theta(t)=\omega_{s} t=2 \pi f_{s} t
$$

Here, the time $t$ is defined by the number of momentum revolutions $n$ in the ring,

$$
t=n \cdot T_{\mathrm{rev}}=\frac{n}{f_{\mathrm{rev}}} .
$$

The spin-precession frequency $f_{s}$, related to $\vec{\Omega}^{\mathrm{Lab}}$ introduced in Eq. (8), can be expressed also via

$$
f_{s}=\frac{\Omega^{\mathrm{Lab}}}{2 \pi}=\frac{G \gamma}{\cos \xi_{\mathrm{EDM}}} \cdot f_{\mathrm{rev}}=\nu_{s} \cdot f_{\mathrm{rev}},
$$

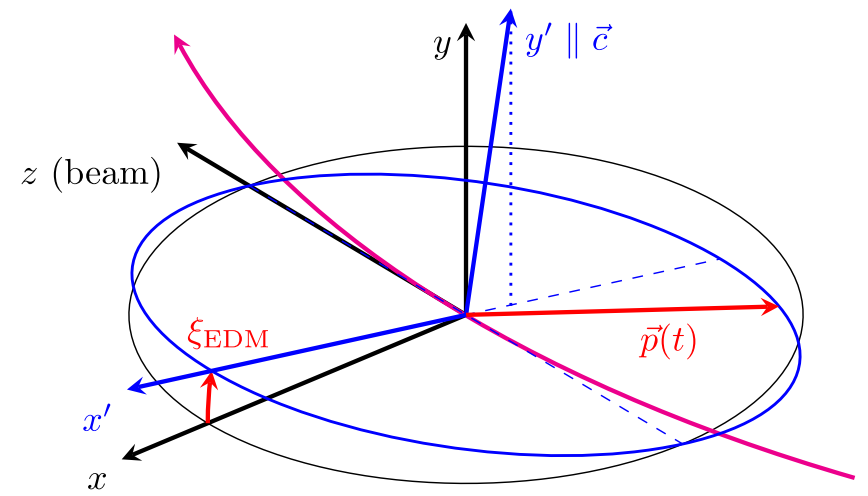

FIG. 1. The beam particles move along the $z$ direction. In the presence of an EDM, i.e., $\xi_{\mathrm{EDM}}>0$, the spins precess around the $\vec{c}$ axis, and an oscillating vertical polarization component $p_{y}(t)$ is generated, as shown in Fig. 2.

where $f_{\text {rev }}$ denotes the revolution frequency, and $\nu_{s}$ the spin tune, i.e., the number of spin precessions per turn in the ring. A negative $G$ factor indicates that the precession proceeds opposite to the orbit revolution.

Thus, a one-turn matrix for the ring including the EDM effect is obtained by inserting $\theta(t)$ from Eq. (15) into Eq. (12) at $t=T_{\text {rev }}=1 / f_{\text {rev }}$. For comparison with numerical simulations, the ring matrix is explicitly given below (to four decimal places) using the parameters listed in Table I,

$$
\begin{aligned}
& \mathbf{U}_{\text {ring }}\left(\vec{c}, T_{\text {rev }}\right) \\
& =\left(\begin{array}{ccc}
5.3063 \times 10^{-1} & -1.4333 \times 10^{-6} & -8.4760 \times 10^{-1} \\
-1.4333 \times 10^{-6} & 1.0000 & -2.5883 \times 10^{-6} \\
8.4760 \times 10^{-1} & 2.5883 \times 10^{-6} & 5.3063 \times 10^{-1}
\end{array}\right) .
\end{aligned}
$$

\section{E. Polarization evolution in the ring}

The evolution of the polarization vector $\vec{S}_{1}$ as a function of time in the ideal bare ring is then described by

$$
\vec{S}_{1}(t)=\mathbf{U}_{\text {ring }}(\vec{c}, t) \times \vec{S}_{0},
$$

where $\vec{S}_{0}$ denotes the initial polarization vector. Throughout the present paper, the single-particle spin evolution is described by unitary transformations which preserve the magnitude of the polarization.

Figure 1 shows the situation when the spin rotation axis $\vec{c}$, defined by Eq. (14), is tilted with respect to the normal to the ring plane $\vec{n}$ ( $y$ axis in the figure).

In Fig. 2, the solutions of $\vec{S}_{1}(t)$ from Eq. (19) for two different initial in-plane polarization vectors $\vec{S}_{0}$ are shown

\footnotetext{
${ }^{5}$ Here, it is supposed that the polarimeter is ideally aligned to the physical ring plane so that the left-right asymmetry measures $p_{y}(t)$, and the up-down asymmetry measures $p_{x}(t)$.
} 

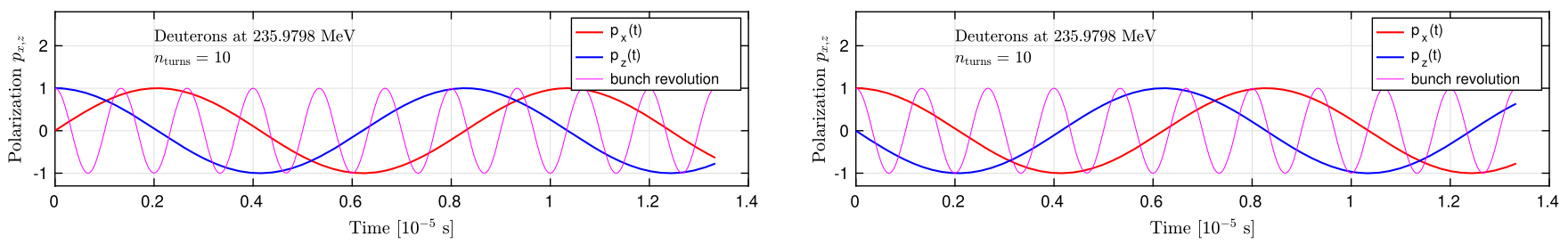

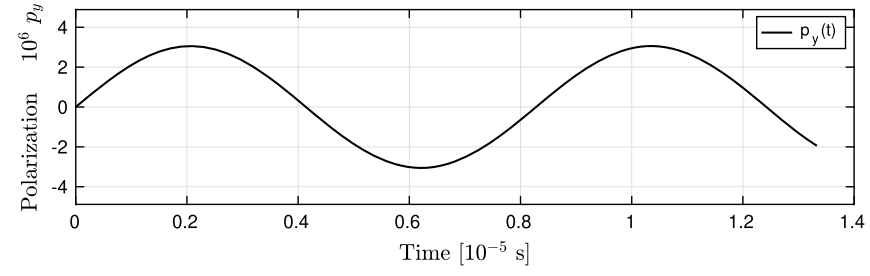

(a)

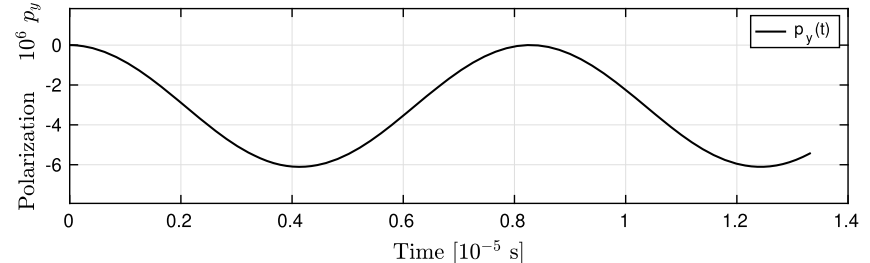

(b)

FIG. 2. Polarization evolution during idle precession for ten turns in an ideal ring using Eq. (19) and the parameters listed in Table I. Panel (a) shows $p_{x}(t), p_{z}(t)$ and $p_{y}(t)$ for an initially longitudinal polarization, and panel (b) the same for initial sideways polarization. The bunch revolution is indicated as well. The magnitude of the $p_{y}$ oscillation amplitude is equal to the tilt angle $\xi_{\mathrm{EDM}}$ [see also Eq. (14) and Fig. 1].

for ten turns. It is visible that the polarization evolution occurs counterclockwise with respect to the clockwise rotation of the particles in the ring, since the deuteron $G$ factor is negative.

\section{III. rf WIEN FILTER IN A RING}

\section{A. Electric and magnetic fields of the rf Wien filter}

The rf Wien filter, described in [4], has been designed in order to be able to manipulate the spins of the stored particles, avoiding as much as possible effects on the beam orbit. To this end, great care was taken to minimize the unwanted field components of the Wien filter and to characterize them via the polynomial chaos expansion [5]. In the EDM mode, the main component of the magnetic induction $\vec{B}^{\mathrm{WF}}$ is oriented along the $y$ axis, and the main component of the electric field $\vec{E}^{\mathrm{WF}}$ along the $x$ axis.

In order to avoid betatron oscillations in the beam, the magnetic and electric fields must be matched to each other to provide a vanishing Lorentz force $\vec{F}_{\mathrm{L}}$ [see Eq. (3) of [4] ],

$$
\vec{F}_{\mathrm{L}}=0 \Leftrightarrow \vec{E}_{x}^{\mathrm{WF}}+c \vec{\beta} \times \vec{B}_{y}^{\mathrm{WF}}=0
$$

According to a full-wave simulation (FWS), ${ }^{6}$ including the ferrite cage (see label 6 in Fig. 1 of [4]), for an input power of $1 \mathrm{~kW}$, a field integral of $\vec{B}^{\mathrm{WF}}$ along the beam axis of

$$
\int_{-\ell_{\mathrm{WF}} / 2}^{\ell_{\mathrm{WF}} / 2} \vec{B}^{\mathrm{WF}} \mathrm{d} z=\left(\begin{array}{c}
2.73 \times 10^{-9} \\
2.72 \times 10^{-2} \\
6.96 \times 10^{-7}
\end{array}\right) \mathrm{T} \mathrm{mm}
$$

\footnotetext{
${ }^{6}$ The full-wave simulations (FWS) have been carried out using CST Microwave Studio, Computer Simulation Technology AG, Darmstadt, Germany, http://www.cst.com.
}

is obtained. Here, the active length of the rf Wien filter [4], denoted by

$$
\ell_{\mathrm{WF}}=1550 \mathrm{~mm}
$$

is defined as the region, where the fields are nonzero. Under these conditions, the corresponding integrated electric field components with ferrites are

$$
\int_{-\ell_{\mathrm{WF}} / 2}^{\ell_{\mathrm{WF}} / 2} \vec{E}^{\mathrm{WF}} \mathrm{d} z=\left(\begin{array}{r}
3324.577 \\
0.018 \\
0.006
\end{array}\right) \mathrm{V}
$$

The design and construction of the rf Wien filter includes a ferrite cage surrounding the electrodes, which improves the field homogeneity and increases the magnitude of the fields [4]. However, in order to simplify the installation, the rf Wien filter was installed at COSY without ferrites. In addition, it was decided to proceed without ferrites until a first direct deuteron EDM measurement is available.

For this situation without ferrites, and for an input power of $1 \mathrm{~kW}$ [ignoring the unwanted components of the field integrals $\left(B_{x}^{\mathrm{WF}}, B_{z}^{\mathrm{WF}}\right.$, and $\left.\left.E_{y}^{\mathrm{WF}}, E_{z}^{\mathrm{WF}}\right)\right]$, one obtains from the full-wave simulation (FWS)

$$
\begin{aligned}
\mathrm{EDL}_{x}^{\mathrm{FWS}} & =\int_{-\ell_{\mathrm{WF}} / 2}^{\ell_{\mathrm{WF}} / 2} E_{x}^{\mathrm{WF}} \mathrm{d} z=2204.677323 \mathrm{~V}, \quad \text { and } \\
\mathrm{BDL}_{y}^{\mathrm{FWS}} & =\int_{-\ell_{\mathrm{WF}} / 2}^{\ell_{\mathrm{WF}} / 2} B_{y}^{\mathrm{WF}} \mathrm{d} z=1.598492 \times 10^{-5} \mathrm{~T} \mathrm{~m} .
\end{aligned}
$$

The ratio of electric and magnetic field integrals from the FWS yields 
TABLE II. Values for the main electric and magnetic field integrals from the full wave simulation with and without ferrites for an input power of $1 \mathrm{~kW}$ where $\vec{B}^{\mathrm{WF}} \| \vec{e}_{y}$. The last column lists the electric and magnetic field integrals of an idealized Wien filter used in the simulations. In this case, the unwanted field components vanish, i.e., $\int E_{y}^{\mathrm{WF}} \mathrm{d} z=\int E_{z}^{\mathrm{WF}} \mathrm{d} z=\int B_{x}^{\mathrm{WF}} \mathrm{d} z=\int B_{z}^{\mathrm{WF}} \mathrm{d} z=0$.

\begin{tabular}{lcrr}
\hline \hline & & \multicolumn{2}{c}{ Without ferrites } \\
\cline { 3 - 4 } Field integrals rf Wien filter & With ferrites (Real WF) & (Real WF) & (Idealized Wien filter) \\
\hline $\int E_{x}^{\mathrm{WF}} \mathrm{d} z[\mathrm{~V}]$ & $3.325 \times 10^{3}$ & $2.204677 \times 10^{3}$ & $2.20000000 \times 10^{3}$ \\
$\int B_{y}^{\mathrm{WF}} \mathrm{d} z[\mathrm{~T} \mathrm{~m}]$ & $2.720 \times 10^{-5}$ & $1.598492 \times 10^{-5}$ & $1.59749820 \times 10^{-5}$ \\
\hline \hline
\end{tabular}

$$
\frac{1}{\beta c} \cdot \frac{\mathrm{EDL}_{x}^{\mathrm{FWS}}}{\mathrm{BDL}_{y}^{\mathrm{FWS}}}=1.0015
$$

and should ideally be equal to unity. The subsequent calculations use the field integrals of an idealized Wien filter with vanishing Lorentz force $\vec{F}_{\mathrm{L}}$, given in the last column of Table II.

A field amplification factor is applied in the simulations to increase the field integrals of the ideal rf Wien filter (last column Table II) in the simulations, so that

$$
\begin{aligned}
& \left.\int E_{x}^{\mathrm{WF}} \mathrm{d} z\right|_{\text {used }}=\left.f_{\text {ampl }} \cdot \int E_{x}^{\mathrm{WF}} \mathrm{d} z\right|_{\text {ideal }} \\
& \left.\int B_{y}^{\mathrm{WF}} \mathrm{d} z\right|_{\text {used }}=\left.f_{\text {ampl }} \cdot \int B_{y}^{\mathrm{WF}} \mathrm{d} z\right|_{\text {ideal }} .
\end{aligned}
$$

The field amplification allows one to speed up the simulation calculations accordingly, without affecting other aspects of the spin dynamics of the polarization evolution in the ring. The reason is that the involved spin rotations scale with the field integrals applied by the rf Wien filter. It should be noted that if instead the magnitude of the EDM would be scaled up, the spin dynamics would be affected because $\xi_{\mathrm{EDM}}$ becomes larger (see Fig. 1). In the description of the spin evolution via spin rotations on the closed orbit, momentum and position kicks are not considered.

\section{B. Rotations induced by the rf Wien filter}

The effect of the rf Wien filter on the polarization evolution in the ring is implemented by an additional rotation matrix. The spin rotation in the Wien filter depends on the applied field integrals (right column of Table II), multiplied by the factor $f_{\text {ampl }}$.

\section{Spin rotation angle in the Wien filter}

In the following, the spin rotation angle $\psi_{\mathrm{WF}}$ in the $\mathrm{rf}$ Wien filter is calculated numerically using the ThomasBMT equation of Eqs. (1) and (2) with $\vec{\Omega}^{\mathrm{EDM}}=0$. We start with an initial spin vector

$$
\vec{S}_{\text {in }}=\left(\begin{array}{l}
0 \\
0 \\
1
\end{array}\right)
$$

and we compute the final polarization vector $\vec{S}_{\text {fin }}$ via

$$
\frac{\Delta \vec{S}}{\Delta t}=\frac{\vec{S}_{\mathrm{fin}}-\vec{S}_{\mathrm{in}}}{\Delta t}=\vec{\Omega}^{\mathrm{MDM}} \times \vec{S}_{\mathrm{in}}
$$

Electric and magnetic field vectors for $\vec{\Omega}^{\mathrm{MDM}}$ in Eq. (2) are obtained by computing the average fields from the idealized field integrals of the $\mathrm{rf}$ Wien filter (last column of Table II), given by

$$
\begin{aligned}
& \vec{E}^{\mathrm{WF}}=\left(\begin{array}{c}
\frac{\int E_{x}^{\mathrm{WF}} \mathrm{d} z}{\ell_{\mathrm{WF}}} \\
0 \\
0
\end{array}\right), \quad \text { and } \\
& \vec{B}^{\mathrm{WF}}=\left(\begin{array}{c}
0 \\
\frac{\int B_{y}^{\mathrm{WF} \mathrm{d} z}}{\ell_{\mathrm{WF}}} \\
0
\end{array}\right),
\end{aligned}
$$

where the effective length of the Wien filter is taken from Eq. (22). These conditions provide for a vanishing Lorentz force $\vec{F}_{\text {L }}$ [see also Eq. (20)].

After passing the rf Wien filter once, the final polarization vector is given by

$$
\begin{aligned}
\vec{S}_{\mathrm{fin}} & =\left(\vec{\Omega}^{\mathrm{MDM}} \times \vec{S}_{\mathrm{in}}\right) \cdot \Delta t+\vec{S}_{\mathrm{in}} \\
& \approx\left(\vec{\Omega}^{\mathrm{MDM}} \times \vec{S}_{\mathrm{in}}\right) \cdot \frac{\ell_{\mathrm{WF}}}{\beta c}+\vec{S}_{\mathrm{in}},
\end{aligned}
$$

and, after normalizing $\vec{S}_{\text {fin }}$ to unity, the angle between $S_{\text {in }}$ and $\vec{S}_{\text {fin }}$ is determined from the four-quadrant inverse tangent

$$
\arctan 2\left(\vec{S}_{\text {in }} \times \vec{S}_{\text {fin }}, \vec{S}_{\text {in }} \cdot \vec{S}_{\text {fin }}\right)=\left(\begin{array}{c}
0.000000 \\
\psi_{\mathrm{WF}} \\
0.000000
\end{array}\right),
$$

yielding numerically

$$
\left|\psi_{\mathrm{WF}}\right|=3.75845773 \times 10^{-6} \mathrm{rad}
$$

using the parameters listed in Table I. 
The spin-rotation angle in the rf Wien filter, divided by the idealized transverse magnetic field integral from Table II, yields

$$
\frac{\left|\psi_{\mathrm{WF}}\right|}{\int B_{y}^{\mathrm{WF}} \mathrm{d} z}=2.35271485 \times 10^{-1} \operatorname{rad~T}^{-1} \mathrm{~m}^{-1}
$$

Validating the numerical result for the spin-rotation angle $\psi_{\mathrm{WF}}$ in the rf Wien filter obtained in Eq. (32) against the analytic expression, given in Eq. (13) of [18], yields

$$
\begin{aligned}
\Omega_{\mathrm{WF}} \cdot \Delta t=\psi_{\mathrm{WF}} & =-\frac{q}{m} \cdot \frac{(1+G)}{\gamma^{2}} \cdot B^{\mathrm{WF}} \cdot \frac{\ell_{\mathrm{WF}}}{\beta c} \\
& =-\frac{q}{m} \cdot \frac{(1+G)}{\gamma^{2} \beta c} \int B_{\perp} \mathrm{d} \ell \\
& =-\frac{q}{m} \cdot \frac{(1+G)}{\gamma^{2} \beta^{2} c^{2}} \int E_{\perp} \mathrm{d} \ell \\
& =-3.75845773 \times 10^{-6} \mathrm{rad},
\end{aligned}
$$

where the time interval $\Delta t$ in the Wien filter has been expressed through the length $\ell_{\mathrm{WF}}$.

The spin rotation angle in the rf Wien filter, given in Eq. (34), constitutes an upper limit, which corresponds to a situation when a sharp $\delta$-function-like bunch passes through the device. Realistically, the bunch distribution has to be folded in, and the average spin-rotation angle will be reduced correspondingly.

\section{2. $r f$ Wien filter rotation matrix}

The spin-rotation angle of the rf Wien filter changes as a function of time according to

$$
\psi(t)=\psi_{\mathrm{WF}} \cos \left(\omega_{\mathrm{WF}} \cdot t+\phi_{\mathrm{rf}}\right)
$$

where

$$
\omega_{\mathrm{WF}}=2 \pi f_{\mathrm{WF}} .
$$

The parametric spin resonance condition for the Wien filter frequency is given by the sum of a harmonic multiple $(K)$ of the revolution frequency $f_{\text {rev }}$ and the spin-precession frequency $f_{s}$ [Eq. (17)],

$$
\begin{aligned}
f_{\mathrm{WF}} & =K \cdot f_{\mathrm{rev}}+f_{s} \\
& =\left(K+\frac{G \gamma}{\cos \xi_{\mathrm{EDM}}}\right) \cdot f_{\mathrm{rev}}, \quad K \in \mathbb{Z} .
\end{aligned}
$$

The rf Wien filter was designed to allow for the operation at different frequencies $f_{\mathrm{WF}}$ [4]. Figure 3 shows the available harmonics (closed circles) for protons (at $T=135 \mathrm{MeV}$ ) and for deuterons (at $P=970 \mathrm{MeV} / c$ ).

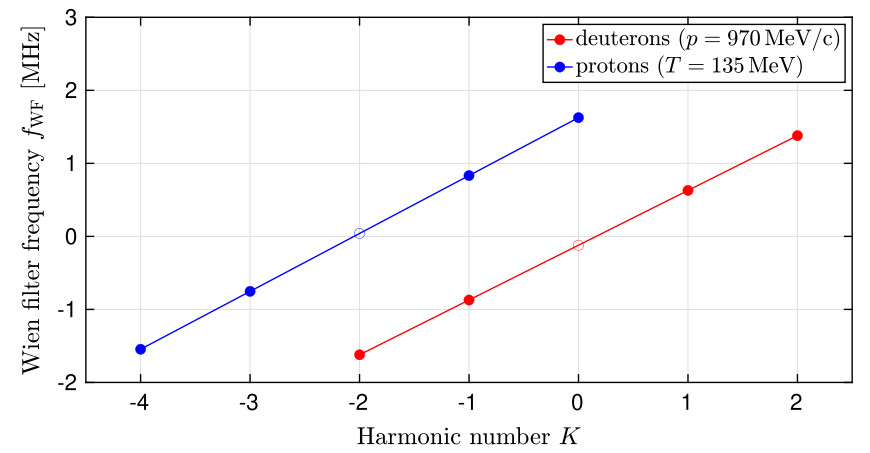

FIG. 3. Frequencies of the rf Wien filter for different harmonics for proton (at $T=135 \mathrm{MeV}$ ) and deuterons from Eq. (37). The frequencies shown by open symbols (deuterons at $K=0$, and protons at $K=-2$ ) are not reachable with the present setup of the driving circuit of the rf Wien filter.

The rf Wien filter rotation matrix is given by

$$
\mathbf{U}_{\mathrm{WF}}(t)=\mathbf{R}\left[\vec{n}_{\mathrm{WF}}, \psi(t)\right]
$$

where in the generic case, $\vec{n}_{\mathrm{WF}}$ is a unit vector along the magnetic field of the Wien filter. The case

$$
\vec{n}_{\mathrm{WF}}=\vec{e}_{y}
$$

for instance, denotes the Wien filter EDM mode. The rf Wien filter matrix $\mathbf{U}_{\mathrm{WF}}(t)$ is only evaluated once per turn when the condition

$$
\bmod \left(t, T_{\text {rev }}\right) \equiv 0
$$

is met stroboscopically, otherwise, the implemented function returns the $\mathbf{I}_{3}$ unit matrix.

When the Wien filter is rotated around the beam axis $(z)$ by some angle $\phi_{\mathrm{rot}}^{\mathrm{WF}}$, and

$$
\begin{aligned}
\vec{n}_{\mathrm{WF}} & =\vec{n}_{\mathrm{WF}}\left(\phi_{\mathrm{rot}}^{\mathrm{WF}}\right)=\mathbf{R}\left(\vec{e}_{z}, \phi_{\mathrm{rot}}^{\mathrm{WF}}\right) \times \vec{e}_{y} \\
& =\left(\begin{array}{ccc}
\cos \left(\phi_{\mathrm{rot}}^{\mathrm{WF}}\right) & -\sin \left(\phi_{\mathrm{rot}}^{\mathrm{WF}}\right) & 0 \\
\sin \left(\phi_{\mathrm{rot}}^{\mathrm{WF}}\right) & \cos \left(\phi_{\mathrm{rot}}^{\mathrm{WF}}\right) & 0 \\
0 & 0 & 1
\end{array}\right) \times \vec{e}_{y},
\end{aligned}
$$

the oscillations also receive a contribution from the rotation of the MDM in the horizontal magnetic field.

\section{Polarization evolution in the ring with rf Wien filter}

The evolution of the polarization vector $\vec{S}$ as a function of time $t$ in the ring with rf Wien filter can be numerically evaluated via 


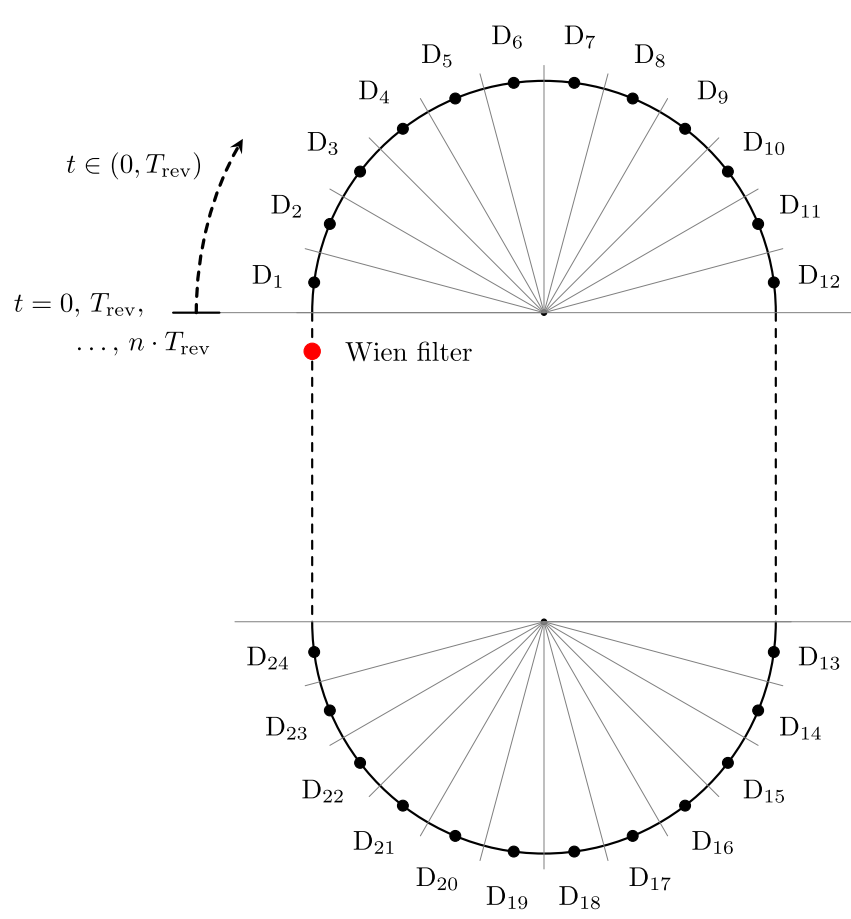

FIG. 4. Sequence of elements in the ring, corresponding to Eq. (42). The $\mathrm{D}_{i}(i=1, \ldots, 24)$ indicate the 24 dipole magnets of COSY. The counting of $t$ begins with one turn in the ring, and, as indicated, the Wien filter is passed at the end of each revolution. For the discussion presented here, the dashed lines have zero length.

$$
\begin{aligned}
\vec{S}_{2}(t)= & \underbrace{\mathbf{U}_{\text {ring }}\left(\vec{c}, t-n \cdot T_{\text {rev }}\right)}_{\text {rest of last turn }} \\
& \times \underbrace{\left[\mathbf{U}_{\mathrm{WF}}\left(t=n \cdot T_{\text {rev }}\right) \times \mathbf{U}_{\text {ring }}\left(\vec{c}, T_{\text {rev }}\right)\right]}_{\text {turn n }} \cdots \\
& \times \underbrace{\left[\mathbf{U}_{\mathrm{WF}}\left(t=2 \cdot T_{\text {rev }}\right) \times \mathbf{U}_{\text {ring }}\left(\vec{c}, T_{\text {rev }}\right)\right]}_{\text {turn } 2} \\
& \times \underbrace{\left[\mathbf{U}_{\mathrm{WF}}\left(t=T_{\text {rev }}\right) \times \mathbf{U}_{\text {ring }}\left(\vec{c}, T_{\text {rev }}\right)\right]}_{\text {turn } 1} \times \vec{S}_{0} .
\end{aligned}
$$

The corresponding situation of the ring is illustrated in Fig. 4. The spin rotations in the ring can be described by $\mathbf{U}_{\text {ring }}$. A turn begins with the revolution in the ring at $t=0$, $T_{\text {rev }}, \ldots, n \cdot T_{\text {rev }}$, and it ends with one pass through the rf Wien filter. Between two successive points in time at which a particle encounters the rf Wien filter, its spin is just idly precessing in the machine. Sequential unitary rotations in Eq. (42) manifestly preserve the magnitude of the polarization.

According to Eq. (42), the spin motion is stroboscopic in the sense that the spin rotation follows the angle $\psi(t)$ of the rf Wien filter [Eq. (35)] turn by turn. The rf Wien filter therefore induces a stroboscopic turn-by-turn conversion of the transverse in-plane polarization into a vertical one (or vice versa). In the spirit of Eq. (30), Eq. (42) can be cast in the form of a system of finite difference equations. In the continuous limit, one obtains a system of homogeneous first order differential equations with time-dependent coefficients which are periodic functions of $\omega_{\mathrm{WF}} \cdot t$. The latter property is imposed by the time-dependent spin rotation in the Wien Filter, see Eq. (35).

The transition to the continuous limit is furnished by the Bogolyubov-Krylov-Mitropolsky (BKM) averaging [14], which approximates the turn-by-turn evolution by a continuous dependence on the revolution number, given by $n=f_{\text {rev }} \cdot t$ [Eq. (16)]. Then, Eq. (37) provides the condition for exact parametric resonance [20-22] that generates the up-down oscillation of the polarization. For the generic orientation of the rf Wien filter, the BKM averaged buildup of the vertical polarization proceeds with the resonance tune (or strength) [18]

$$
\varepsilon^{\mathrm{EDM}}=\frac{1}{4 \pi}\left|\vec{c} \times \vec{n}_{\mathrm{WF}}\right| \cdot \psi_{\mathrm{WF}} .
$$

The above formula is universal for $\mathrm{rf}$ spin rotators of all kinds, rf Wien filters, electric and magnetic rf dipoles, and rf solenoids.

In the EDM mode [see Eq. (39)], the above equation yields

$$
\begin{aligned}
\varepsilon^{\mathrm{EDM}} & =\frac{1}{4 \pi} \sin \xi_{\mathrm{EDM}} \cdot \psi_{\mathrm{WF}} \\
& =-9.133154 \times 10^{-13}
\end{aligned}
$$

using the parameters of Table I. Therefore, in the absence of other perturbing spin rotations in the ring, a measurement of the resonance tune constitutes a direct measurement of the EDM. ${ }^{7}$ The generic case when the stable spin axis $\vec{c}$ acquires an additional tilt from in-plane magnetic fields will be discussed in Sec. IV.

The direct simulations using Eq. (42), discussed below, will furnish important cross-checks with respect to the accuracy of the analytic approximations based on the BKM averaging.

\section{Radial magnetic rf field in the Wien filter}

\section{Driven oscillations and resonance strength $\varepsilon^{\mathrm{MDM}}$}

Driven oscillations of the vertical polarization $p_{y}(t)$ can also be induced by the horizontal magnetic field of the rf Wien filter that couples to the deuteron MDM. As an illustration of the principal features of the polarization evolution under exact parametric resonance [see Eq. (37)], we discuss below the situation where the rf Wien filter is operated in the so-called MDM mode with magnetic field along $-\vec{e}_{x}$, i.e., for $\phi_{\text {rot }}^{\mathrm{WF}}=90^{\circ}$, and where the initial polarization $\vec{S}_{0}=-\vec{e}_{y}$.

\footnotetext{
${ }^{7}$ It should be noted that in conjunction with the resonance tune $\varepsilon$, the superscript "EDM" (or "MDM") is reserved for situations when the primary focus is on the EDM (or MDM) effect itself.
} 


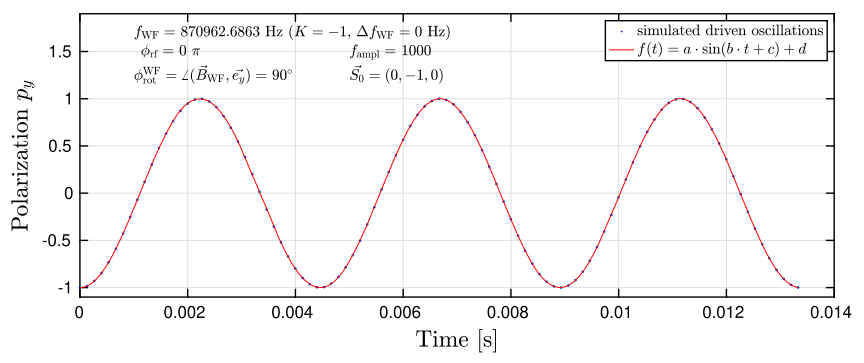

FIG. 5. Simulated driven oscillations on resonance using $\vec{S}_{2}(t)$ from Eq. (42) with initial vertical polarization $\vec{S}_{0}=-\vec{e}_{y}, \phi_{\mathrm{rf}}=0$ [Eq. (37)], and $\phi_{\mathrm{rot}}^{\mathrm{WF}}=90^{\circ}$ [Eq. (41)] for the parameters given in Table I and for the harmonic $K=-1$. The plot contains 101 points for a total of 10000 turns.

In this case $\vec{n}_{\mathrm{WF}}=-\vec{e}_{x}$, and Eq. (43) predicts the resonance strength

$$
\begin{aligned}
\left|\epsilon^{\mathrm{MDM}}\right| & =\frac{\left|\cos \xi_{\mathrm{EDM}} \cdot \psi_{\mathrm{WF}}\right|}{4 \pi} \\
& =2.99088563 \times 10^{-7},
\end{aligned}
$$

where $\psi_{\mathrm{WF}}$ from Eq. (32) was used, and $\xi_{\mathrm{EDM}}$ from Table I.

Using the function for $\vec{S}_{2}(t)$, given in Eq. (42), for the conditions of Table I, driven oscillations for the rf Wien filter with magnetic field aligned along $-\vec{e}_{x}$ [see Eq. (41)] were simulated. One example for $K=-1$ is shown in Fig. 5. Subsequently, the simulated oscillations were fitted using the function

$$
f(t)=p_{y}(t)=a \cdot \sin (b t+c)+d .
$$

The quality of the fit to the numerical data is evaluated in terms of squared deviations via

$$
\mathrm{SSE}=\sum_{i=1}^{n_{\text {points }}} w_{i}\left[p_{y}\left(t_{i}\right)-f\left(t_{i}\right)\right]^{2},
$$

where the weight factors are $w_{i}=1$, and $p_{y}(t)=\vec{e}_{y} \cdot \vec{S}_{2}(t)$. In the last row of Table III, the reduced $\chi^{2}=\mathrm{SSE} / \mathrm{ndf}$ is given, where $n_{\text {points }}=101$, and $\mathrm{ndf}=n_{\text {points }}-4=97$, since the fitted function in Eq. (46) has four parameters.

The fit results are summarized in Table III. The angular velocity $\Omega^{\text {driven }}=b$ was obtained using the field integrals, listed in the right column of Table II. The calculation used a computation time ${ }^{8}$ of about $40 \mathrm{~s}$. Under these conditions, the driven oscillations exactly on resonance have an angular velocity of

$$
\frac{\Omega^{\text {driven }}}{f_{\text {ampl }}}=1.40978 \pm 0.00005 \mathrm{~Hz}
$$

\footnotetext{
${ }^{8}$ All computations, using 64-bit double-precision floating point numbers for which the machine epsilon $=2.2 \times 10^{-16}=$ $2^{-52}$, were performed on a Lenovo T460s machine with i7 Intel (R) Core(TM) i7-6600U CPU@2.60GHz, equipped with 20 GB of RAM.
}

TABLE III. Result of the fit of the driven oscillation on resonance for $K=-1$, shown in Fig. 5, using 10000 turns with 101 data points. The other four cases $K=0,+1$, and \pm 2 , within the given precision, yield identical values. SSE/ndf denotes the sum of squared deviations, computed using Eq. (47), divided by the number of degrees of freedom (ndf).

\begin{tabular}{lc}
\hline \hline$K$ & -1 \\
$f_{\text {ampl }}$ & $10^{3}$ \\
$f_{\mathrm{WF}}$ & $870962.6863 \mathrm{~Hz}$ \\
$a$ & $(10000 \pm 2) \times 10^{-4}$ \\
$b$ & $(1409.7817 \pm 0.0470) \mathrm{s}^{-1}$ \\
$c$ & $(0.4997 \pm 0.0001) \pi$ \\
$d$ & $(0.0000 \pm 0.0001)$ \\
SSE/ndf & $3.801 \times 10^{-7}$ \\
\hline \hline
\end{tabular}

Furthermore, the angular velocity normalized to the real magnetic field integral yields

$\frac{\Omega^{\text {driven }}}{\int B_{y}^{\mathrm{WF}} \mathrm{d} z \cdot f_{\mathrm{ampl}}}=(88.249 \pm 0.003) \mathrm{s}^{-1} \mathrm{~T}^{-1} \mathrm{~m}^{-1}$.

The induced driven oscillations, shown in Fig. 5, correspond to a resonance strength of

$\varepsilon^{\mathrm{MDM}}=\frac{\Omega^{\text {driven }}}{\Omega^{\mathrm{rev}} \cdot f_{\mathrm{ampl}}}=(2.99086 \pm 0.00010) \times 10^{-7}$,

where the factor $f_{\text {ampl }}$ in the denominator corrects for the field enhancement used in the simulation. The resulting value agrees nicely within errors with $\left|\varepsilon^{\mathrm{EDM}}\right|$ of Eq. (45).

\section{Width of the spin resonance}

The detuning of the frequency $f_{\mathrm{WF}}$ at which the rf Wien filter is operated away from the spin-precession frequency $f_{s}$ [see Eqs. (17) and (37)] can be parametrized by substituting in Eq. (36)

$$
f_{\mathrm{WF}} \rightarrow f_{\mathrm{WF}}+\Delta f_{\mathrm{WF}}
$$

From about 50 simulations similar to the one shown in Fig. 6, the oscillation amplitudes and the oscillation frequencies as function of $\Delta f_{\mathrm{WF}}$ are obtained by fitting, invoking again the parametrization given in Eq. (46). In order to reduce the time required for the simulations, a field amplification factor of $f_{\text {ampl }}=10^{3}$ was used, which leads to oscillations that are faster by the same factor. The results, shown in Fig. 7, are corrected for the field amplification factor employed in the simulations. The dependence of the oscillation amplitude $a$ of the simulated data can be described by a Lorentz curve (BreitWigner function) of the form

$$
L\left(\Delta f_{\mathrm{WF}}\right)=h \cdot \frac{\Gamma^{2}}{\Gamma^{2}+\left(2 \Delta f_{\mathrm{WF}}\right)^{2}},
$$




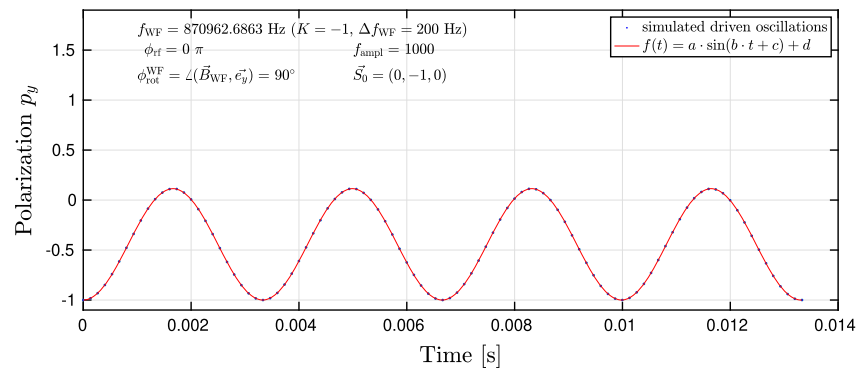

FIG. 6. Off-resonance driven oscillations with $\Delta f_{\mathrm{WF}}=200 \mathrm{~Hz}$ in Eq. (51) for the conditions of Table I.

where

$$
\Delta f_{\mathrm{WF}}=f_{\mathrm{WF}}-f_{s}
$$

The normalization constant and the width of the BreitWigner, obtained from the fit shown in the left panel of Fig. 6, amount to

$$
\begin{aligned}
& h=1.00000 \pm 0.00009 \text { and } \\
& \Gamma=(0.44879 \pm 0.00007) \mathrm{Hz} .
\end{aligned}
$$

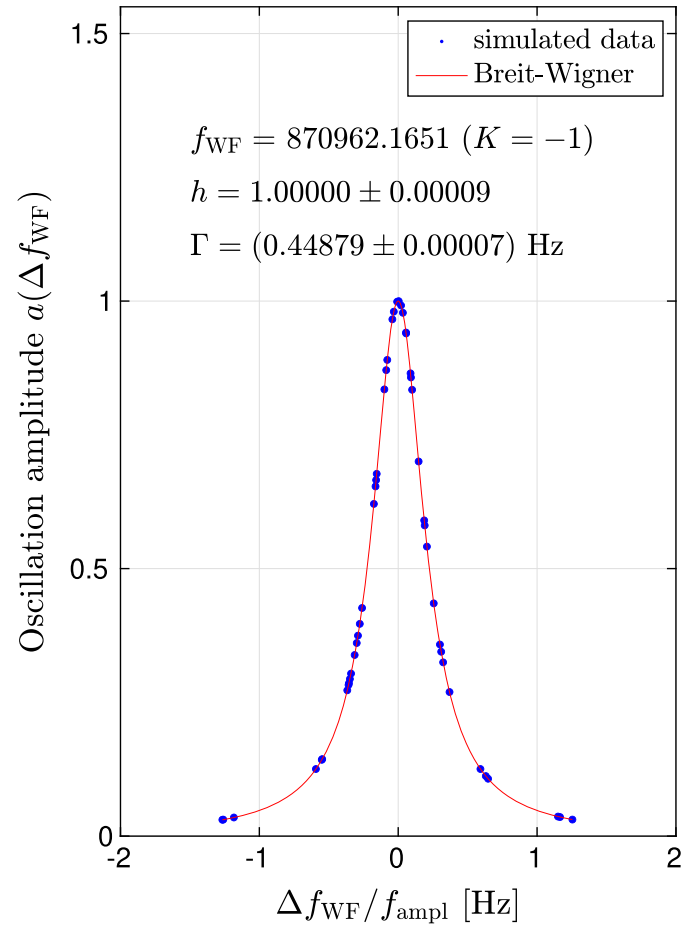

For other harmonic excitations $K=0,1$, and \pm 2 used in the rf Wien filter, within the errors the simulations yield the same results as given above.

It should be emphasized that the off-resonance parametric modulation of the spin motion exhibits a profound difference compared to the more familiar oscillations that are driven by an external harmonic force [20-22]. In parametric resonances, detuning causes a continuously growing phase difference

$$
\Delta \phi_{\mathrm{WF}}=2 \pi \Delta f_{\mathrm{WF}} t
$$

between rf phase and spin-vector phase. The accumulation of the vertical polarization comes to an end as soon as $\Delta \phi_{\mathrm{WF}} \sim \pi / 2$. Evidently, the exact pattern of the buildup of the vertical polarization will depend on the relative magnitude of $\Delta f_{\mathrm{WF}}$ and the buildup frequency $f^{\text {driven }}=\epsilon^{\mathrm{EDM}} f_{\text {rev }}$, as evidenced by comparing Figs. 5 and 6. A full-fledged discussion of the Fourier spectrum of the off-resonance polarization evolution will be reported elsewhere. Here, only the gross features will be illustrated using the previous example when the rf Wien filter is operated in MDM mode, with initial $\vec{S}_{0}(t=0)=-\vec{c} \simeq \vec{e}_{y}$.
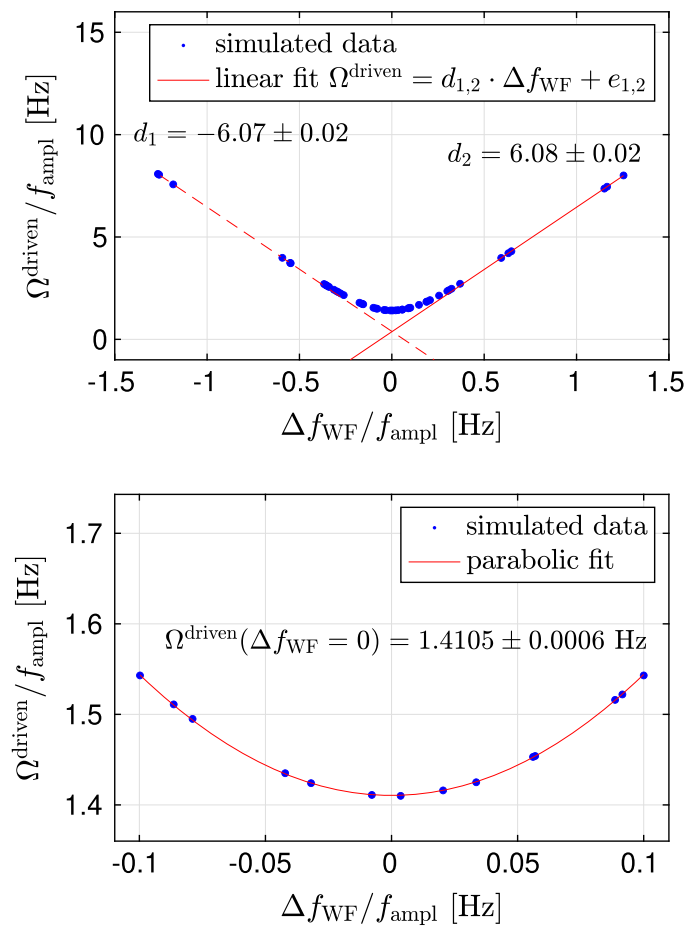

FIG. 7. The left panel shows the amplitude $a$ of simulated driven oscillations as function of the detuned frequency $\Delta f_{\mathrm{WF}}$, corrected for the field amplification factor $f_{\mathrm{ampl}}=10^{3}$ used in the simulations. The oscillation amplitudes $a\left(\Delta f_{\mathrm{WF}}\right)$ were extracted from fits using Eq. (46). The normalization constant $h$ and the full width at half maximum $\Gamma$ of the fitted Breit-Wigner resonance [Eq. (52)] is indicated. The resonance curves for $K= \pm 0, \pm 1$, and \pm 2 are very similar. The panels on the right show the angular velocity $\Omega^{\text {driven }}$ of the oscillations as a function of $\Delta f_{\mathrm{WF}}$, where both axes were corrected for the used $f_{\mathrm{ampl}}$. In the bottom panel on the right, the region of small detuning is shown together with a parabolic fit. The region of large detuning is shown in the top right panel together with linear fits to the six outermost points in each branch. 
The obtained fit results of the simulated oscillations $\Omega^{\text {driven }}\left(\Delta f_{\mathrm{WF}}\right)=b$ [Eq. (46)] are summarized in the panels on the right in Fig. 7. $\Omega^{\text {driven }}$ exhibits a linear dependence on $\Delta f_{\mathrm{WF}}$ at large $\left|\Delta f_{\mathrm{WF}}\right|$ (strong detuning) and a parabolic dependence for weak detuning. The linear fits to the six outermost points, indicated in the top right panel in Fig. 7, show that the transition between the two regimes occurs at about $\Delta f_{\mathrm{WF}} \approx \pm 0.2 \mathrm{~Hz}$.

In the following, the off-resonance results of Fig. 7 are subjected to an interpretation in terms of the BKM averaging. For an exact parametric resonance, the BKM averaging precisely predicts the harmonic evolution

$$
\vec{S}_{y}(t)=-\vec{e}_{y} \cos \left(2 \pi \epsilon^{\mathrm{EDM}} f_{\text {rev }} t\right),
$$

with unity amplitude. In the off-resonance case, the BKM averaging dictates the substitution

$$
t \rightarrow \frac{\sin \left(\pi \Delta f_{\mathrm{WF}} t\right)}{\pi \Delta f_{\mathrm{WF}}} .
$$

The above substitution, together with Eq. (50), leads to

$$
S_{y}(t)=-\cos \left(2 \frac{f^{\text {driven }}}{\Delta f_{\mathrm{WF}}} \sin \left(\pi \Delta f_{\mathrm{WF}} t\right)\right) .
$$

For the limit of strong detuning, $\Delta f_{\mathrm{WF}} \gg f^{\text {driven }}$, one obtains

$$
S_{y}(t) \approx-1+\left(\frac{f^{\text {driven }}}{\Delta f_{\mathrm{WF}}}\right)^{2}\left[1-\cos \left(2 \pi \Delta f_{\mathrm{WF}} t\right)\right] .
$$

In the above expression, the higher harmonics $\cos \left(2 \pi N \Delta_{\mathrm{WF}} t\right)$ are suppressed by powers of the small parameter $\left(f^{\text {driven }} / \Delta f_{\mathrm{WF}}\right)^{2 N}$ and were omitted. The gross features of $S_{y}(t)$ can then be approximated by the harmonic expansion of Eq. (46), yielding

$$
\begin{gathered}
a \simeq\left(\frac{f^{\text {driven }}}{\Delta f_{\mathrm{WF}}}\right)^{2}, \\
b=\Omega^{\text {driven }}\left(\Delta f_{\mathrm{WF}}\right) \simeq 2 \pi \Delta f_{\mathrm{WF}}, \quad \text { and } \\
c \simeq \frac{\pi}{2} .
\end{gathered}
$$

The linear behavior of $\Omega^{\text {driven }}$ at large $\Delta f_{\mathrm{WF}}$, governed by Eq. (61) and depicted in the right top panel of Fig. 7, as expected yields for both branches a slope parameter near $\pm 2 \pi$,

$$
\begin{aligned}
& d_{1}=-6.07 \pm 0.02, \quad \text { and } \\
& d_{2}=6.08 \pm 0.02 .
\end{aligned}
$$

The quadratic fit to $\Omega^{\text {driven }}\left(\Delta f_{\mathrm{WF}}\right)$ in the bottom right panel of Fig. 7 yields

$$
\Omega^{\text {driven }}\left(\Delta f_{\mathrm{WF}}=0\right)=(1.41051 \pm 0.00063) \mathrm{Hz},
$$

which is consistent with the result for the same quantity, given in Eq. (48).

Equating the asymptotic behavior of Eq. (60) to the one of the Breit-Wigner parametrization of Eq. (52),

$$
\left(\frac{f^{\text {driven }}}{\Delta f_{\mathrm{WF}}}\right)^{2} \approx\left(\frac{\Gamma}{2 \Delta f_{\mathrm{WF}}}\right)^{2}
$$

one obtains

$$
\Gamma \simeq \frac{1}{\pi} \Omega^{\text {driven }}\left(\Delta f_{\mathrm{WF}}=0\right)=0.449 \mathrm{~Hz},
$$

which agrees well with the fit result given in Eq. (54).

At weak detuning, the argument of the cosine function in Eq. (58) initially rises linearly with $t$, then reaches a maximum and starts to decrease around

$$
t=T_{\mathrm{BKM}}=\frac{\pi}{2 \Delta f_{\mathrm{WF}}} .
$$

At still larger $t$, the argument is a sine function of time with the period $4 T_{\mathrm{BKM}}$, i.e., it exhibits the beating pattern, typical of a spin echo. In our simulations, the spinevolution time is limited, $t \leq T_{\max }=0.013 \mathrm{~s}$. Evidently, the finite $T_{\max }$ introduces a new frequency scale of

$$
\Delta f_{\min }=\frac{2}{T_{\max } f_{\mathrm{ampl}}} \approx 0.154 \mathrm{~Hz} .
$$

With decreasing $\Delta f_{\mathrm{WF}}$, we expect a change of the dependence of $\Omega^{\text {driven }}$ as a function of $\Delta f_{\mathrm{WF}}$ as soon as the argument of the cosine in Eq. (58) reaches unity. For intermediate detuning, the results shown in Fig. 7 begin to deviate from the linear behavior near a frequency of $\Delta f_{\mathrm{WF}} \approx 0.2 \mathrm{~Hz}$, which roughly agrees with the estimate given in Eq. (68).

The quality factor $Q$ of a parametric spin resonance can be defined as

$$
Q=\frac{f_{s}}{\Gamma},
$$

where $f_{s}$ denotes the spin-resonance frequency at $K=0$ from Eq. (37) and $\Gamma$ is the width of the Breit-Wigner function from Eq. (54). Thus, at a deuteron momentum of $P=970 \mathrm{MeV} / c$, a theoretical estimate of the $Q$ value of the oscillating deuteron spins in the machine amounts to

$$
Q=\frac{120764.751}{0.4488} \approx 270000 .
$$




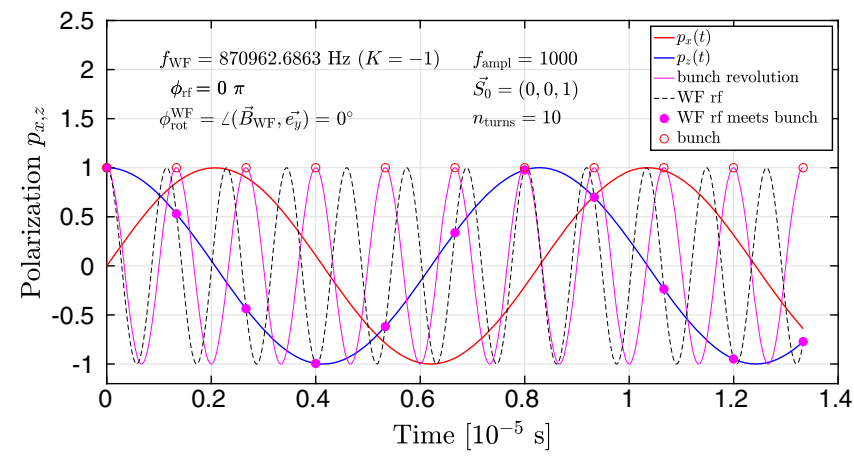

FIG. 8. Horizontal and longitudinal polarization components $p_{x}(t)$ and $p_{z}(t)$ during ten turns in the machine, as described by $S_{2}(t)$ using Eq. (42) for the $K=-1$ harmonic and an initial polarization $\vec{S}_{0}$ in the horizontal ( $x z$ ) plane. The magnetic field $\vec{B}^{\mathrm{WF}}$ of the rf Wien filter points along $\vec{e}_{y}$, and $f_{\text {ampl }}=10^{3}$. Also indicated are bunch revolution and Wien filter rf frequency, and the corresponding rf amplitude when the beam bunch meets the Wien filter rf (pink filled circle).

\section{E. Vertical magnetic field in the rf Wien filter}

With a vertical magnetic field in the rf Wien filter $\left(\vec{n}_{\mathrm{WF}}=\vec{e}_{y}\right)$, in the expression of the spin-resonance strength [Eq. (43)], we obtain

$$
\left|\vec{c} \times \vec{n}_{\mathrm{WF}}\right|=\sin \xi_{\mathrm{EDM}} .
$$

In this situation (EDM mode), the experimental determination of the resonance strength $\varepsilon^{\mathrm{EDM}}$ amounts to the determination of the tilt angle $\xi_{\mathrm{EDM}}$ and of the associated EDM, via Eqs. (11) and (3).

\section{Polarization evolution with development of $p_{y}(t)$}

In the following, the polarization buildup in the machine is addressed. The interplay of the different frequencies involved is illustrated in Fig. 8.

The same situation as in Fig. 8 is depicted in Fig. 9, the only difference is the larger turn number. The graph illustrates the experimental evidence for an EDM, namely a nonvanishing slope of the vertical polarization component $p_{y}(t)$. This slope describes the steady out-of-plane rotation of the polarization vector on the background of oscillations shown in the bottom panels of Fig. 2.

The slope can be determined by fitting using

$$
p_{y}(t)=A \cdot \sin \left(2 \pi f_{s} \cdot t+\phi\right)+B \cdot t+C,
$$

where $f_{s}$ is not a fit parameter, but taken from Eq. (17). The oscillation amplitude $A$ in Fig. 9 perfectly matches the angle $\xi_{\mathrm{EDM}}$, used in the simulation (see Table I). Using the above parametrization, the initial slope is given by

$$
\left.\dot{p}_{y}(t)\right|_{t=0}=B \text {. }
$$

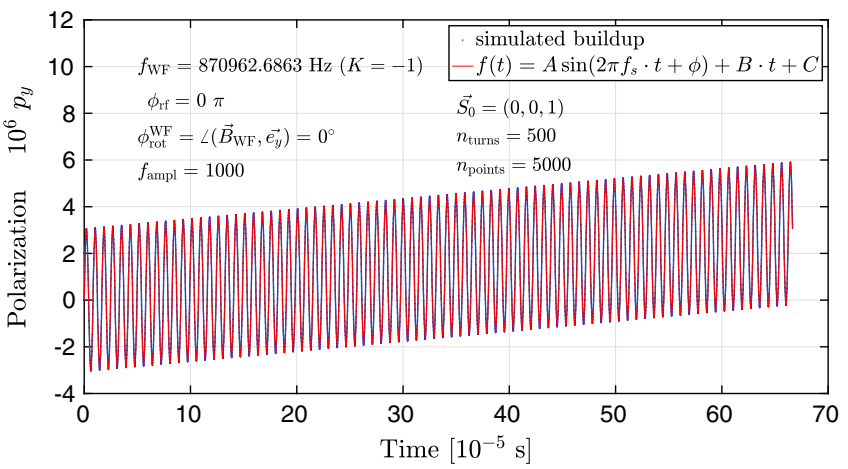

FIG. 9. Buildup of a vertical polarization component for the conditions as indicated. The amplitude of the oscillating $p_{y}(t)$ corresponds to the EDM tilt angle $\xi_{\mathrm{EDM}}$, given in Table I. The red line is a fit to the data using Eq. (72) that yields an initial slope of $\mathrm{d} p_{y}(t) /\left.\mathrm{d} t\right|_{t=0}=B=(4305.059 \pm 5.268) \times 10^{-6} \mathrm{~s}^{-1}$ (for $\left.f_{\text {ampl }}=10^{3}\right)$.

\section{2. $p_{y}(t)$ dependence on the phases $\phi_{\mathrm{rf}}$ and $\phi_{S_{0}^{x}}$}

The rf phase $\phi_{\mathrm{rf}}$ is introduced in Eq. (35). During a real experiment, this phase needs to be maintained by a phase-locking system (for details see [23]). Another way to parametrize the same effect is via the angle $\phi_{S_{0}^{x}}=\angle\left(\vec{S}_{0}, \vec{e}_{x}\right)$, as illustrated in Fig. 10(a), keeping $\phi_{\mathrm{rf}}$ constant.

Within the formalism described in [18], it is the interplay between the stable spin axis $\vec{c}$ at the $\mathrm{rf}$ Wien filter and its magnetic axis $\vec{n}_{\mathrm{WF}}\left(\| \vec{B}^{\mathrm{WF}}\right)$ that controls via $\left[\vec{c} \times \vec{n}_{\mathrm{WF}}\right]$ the dependence on the orientation of $\vec{S}_{0}$. On the other hand, one could start by fixing the orientation of $\vec{S}_{0}$ by picking some angle $\phi_{S_{0}^{x}}$, which amounts to shifting the spin phase while keeping the rf phase fixed. The resulting evolution of $p_{y}(t)$, however, must be the same, except for a possible constant shift between the two phases $\phi_{\mathrm{rf}}$ and $\phi_{S_{0}^{x}}$.

The buildup of a vertical polarization component, which is equivalent to a rotation of the polarization vector out of the ring plane due to the EDM for a set of random azimuthal angles $\phi_{S_{0}^{x}}$ and $\phi_{\mathrm{rf}}$ has been computed. The results are shown in Fig. 11. The fit results are listed in Table IV.

Within the given uncertainties, the two simulated data sets for $\phi_{S_{0}^{x}}$ and $\phi_{\mathrm{rf}}$, as expected, yield the same results. The only difference is a phase shift of $\pi / 2$ between $f\left(\phi_{S_{0}^{x}}\right)$ and $g\left(\phi_{\mathrm{rf}}\right)$, as evidenced by the difference of the parameters $b_{1}-b_{2}$ from Table IV. The weights that are used to find the optimum parameters are all equal for each point of the two data sets.

Correcting the initial slope parameter $a$ in Table IV for the employed field amplification factor used in the simulation yields a prediction for the initial slope that one would expect in an ideal ring in the presence of an EDM of $d=10^{-20} e \mathrm{~cm}$. For an initial polarization $\left|\vec{S}_{0}\right|=1$, with 


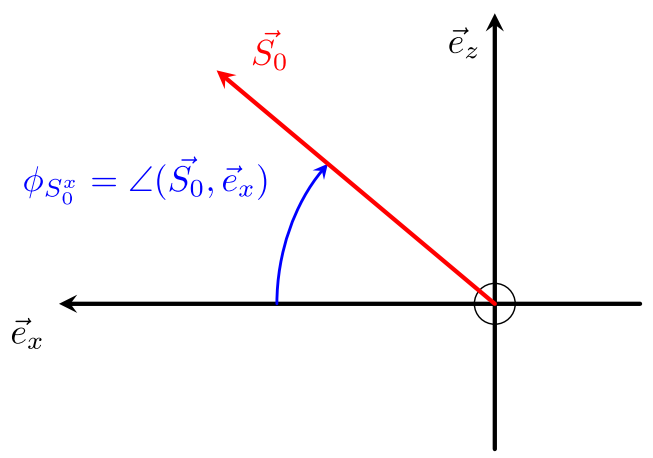

(a)

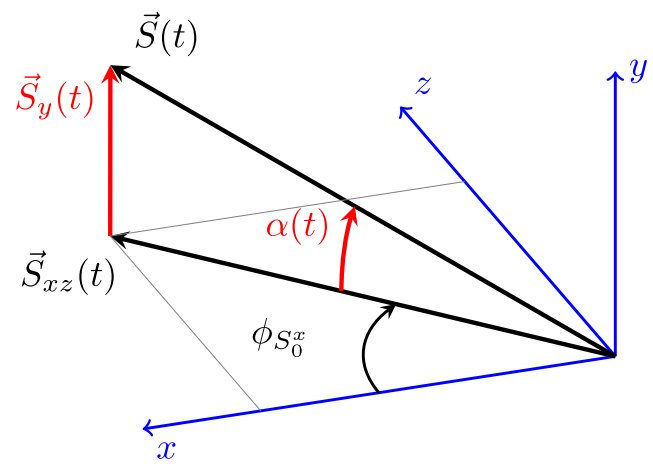

(b)

FIG. 10. Panel (a): Definition of the in-plane initial spin orientation angle $\phi_{S_{0}^{x}}$, and (b) relation between $\vec{S}_{y}(t)$ and the out-of-plane inclination angle $\alpha(t)$.

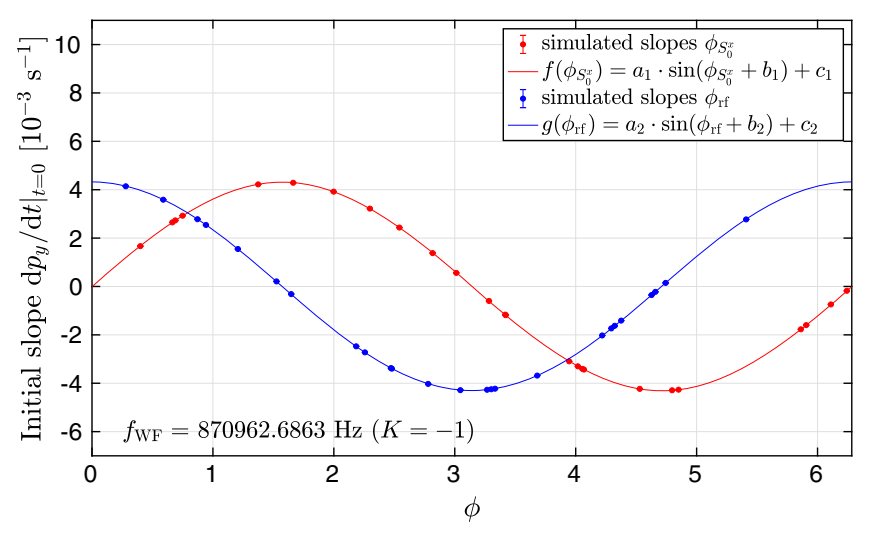

FIG. 11. The red (blue) curve shows the initial slope as a function of 25 random values of $\phi_{S_{0}^{x}}$ and $\phi_{\mathrm{rf}}$, using a field amplification factor $f_{\text {ampl }}=10^{3}$. The simulated data are fitted using the functions indicated in the inset. The resulting parameters are listed in Table IV. Each data point is obtained from a graph like the one shown in Fig. 9, but for 10000 turns and 501 points.

the parameters for the idealized rf Wien filter, given in the last column of Table II, one obtains

$\left.\dot{p}_{y}(t)\right|_{t=0}=\frac{a\left(\phi_{S_{0}^{x}}\right)}{f_{\mathrm{ampl}}}=(4.305 \pm 0.002) \times 10^{-6} \mathrm{~s}^{-1}$.

TABLE IV. Summary of parameters obtained (for $K=-1$ ) via fitting the oscillatory patterns of the initial slopes shown in Fig. 11 as function of $\phi_{S_{0}^{x}}$ and $\phi_{\mathrm{rf}}$, still including the factor $f_{\text {ampl }}=10^{3}$. For the other harmonics $(K=0,1$, and \pm 2$)$, within the given uncertainties, the same values are obtained.

\begin{tabular}{lcc}
\hline \hline & $\phi_{\mathrm{rf}}$ & $\phi_{S_{0}^{x}}$ \\
\hline$a_{1,2}$ & $(4309.884 \pm 2.945) \times 10^{-6}$ & $(4304.623 \pm 2.290) \times 10^{-6}$ \\
$b_{1,2}$ & $(15711.584 \pm 6.254) \times 10^{-4}$ & $(-17.686 \pm 3.637) \times 10^{-4}$ \\
$c_{1,2}$ & $(8.516 \pm 2.075) \times 10^{-6}$ & $(0.367 \pm 1.280) \times 10^{-6}$ \\
$\frac{\chi^{2}}{\text { ndf }}$ & $4.2 \times 10^{-17}$ & $5.9 \times 10^{-17}$ \\
\hline \hline
\end{tabular}

The comparison of $\left.\dot{p}_{y}(t)\right|_{t=0}$ with experiment requires knowledge about the magnitude of $\vec{S}(t)$. The approach taken in [24] appears convenient, because the out-of-plane rotation angle $\alpha$ is independent of the magnitude of the beam polarization. The quantity of interest, indicated in Fig. 10(b), in that case is $\left.\dot{\alpha}(t)\right|_{t=0}$. The polarimeter measures $p_{y}(t)$, irrespective of the in-plane polarization $p_{x z}(t)$, given by

$$
p_{x z}(t)=\sqrt{p_{x z}(0)^{2}-p_{y}(t)^{2}} .
$$

From this it follows that

$\dot{\alpha}(t)=\left.\frac{\mathrm{d}}{\mathrm{d} t} \arctan \left[\frac{p_{y}(t)}{p_{x z}(t)}\right] \Rightarrow \dot{\alpha}(t)\right|_{t=0}=\frac{\left.\dot{p}_{y}(t)\right|_{t=0}}{p_{x z}(0)}$

\section{Initial slope versus slow oscillation}

Figure 12(a) shows the initial slopes for four different assumed EDMs, for an ideal ring and an idealized Wien filter, based on the conditions listed in Table I. The EDMs manifest themselves twofold, namely in different slopes and in larger amplitudes of the fast oscillation. The linear slopes in Fig. 12(a) of course reflect just the very beginning of a sinusoidal oscillation that becomes visible only when the EDM is large. Such a situation is depicted in Fig. 12(b), where

$$
d=10^{-15} e \mathrm{~cm}
$$

has been used in the simulation.

The initial slope of the vertical polarization component is related to the strength of the EDM spin resonance. Another way to obtain this information is to vary the rf phase $\phi_{\mathrm{rf}}$, as indicated in Fig. 11. The initial slope can of course also be obtained from the slow oscillation. The slope can be described by 


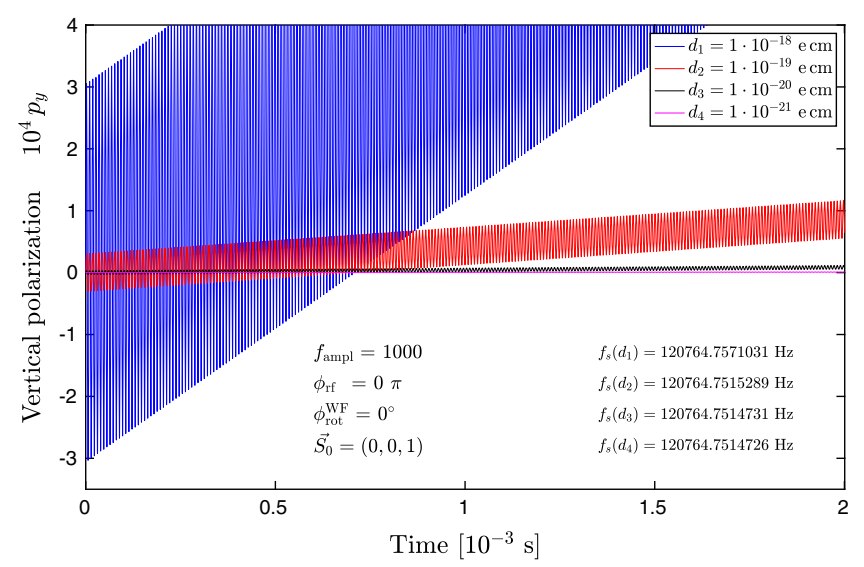

(a)

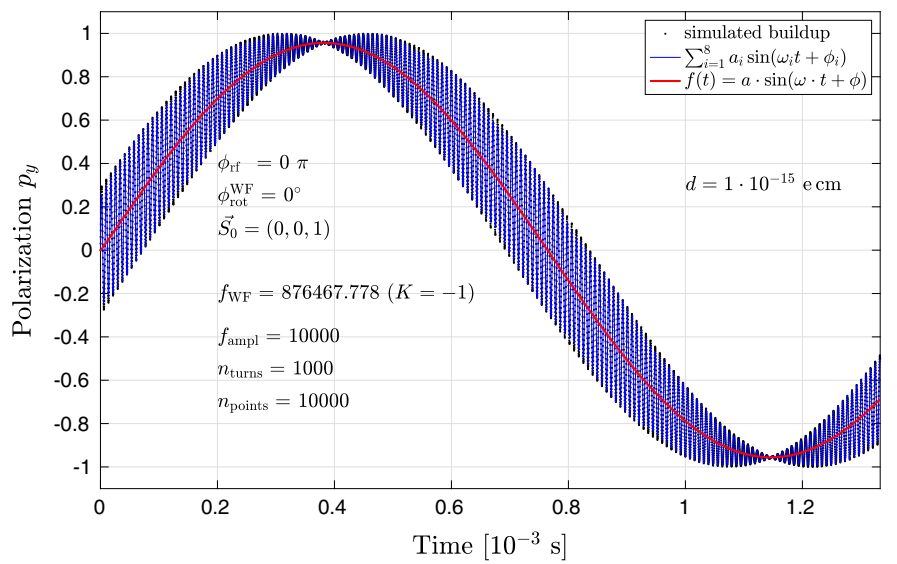

(b)

FIG. 12. Various EDM induced oscillation pattern for short [panel (a)] and long evolution times (b) using different amplification factors and values for the EDM.

$$
p_{y}(t)=a \sin (\omega t) \cdot \cos \phi_{\mathrm{rf}},
$$

which respects the property that for any $\phi_{\mathrm{rf}},\left.p_{y}(t)\right|_{t=0}=0$. The derivative of $p_{y}(t)$ with respect to time is

$$
\begin{aligned}
\dot{p}_{y}(t) & =a \omega \cos (\omega t) \cdot \cos \phi_{\mathrm{rf}} \\
\left.\Rightarrow \dot{p}_{y}(t)\right|_{t=0} & =a \omega \cdot \cos \phi_{\mathrm{rf}}=(3933 \pm 19) \mathrm{s}^{-1},
\end{aligned}
$$

where the value given corresponds to the situation shown in Fig. 12(b).

Numerically, the red curve in Fig. 12(b) has been parametrized by the function

$$
f(t)=p_{y}(t)=a \sin (\omega \cdot t+\phi) .
$$

The amplitude of the averaged oscillation [red curve in Fig. 12(b)] can be predicted directly from the tilt angle of the stable spin axis due to the EDM, via

$$
a=\cos \left(\xi_{\mathrm{EDM}}\left(d=10^{-15} e \mathrm{~cm}\right)\right)=0.9564 .
$$

With $\xi_{\mathrm{EDM}}\left(d=10^{-15} e \mathrm{~cm}\right)=-0.296373$, within the errors, one obtains a perfect match to the value of $a$ given obtained from the fit using Eq. (80), which yields

$$
\begin{aligned}
a & =0.9560 \pm 0.0038, \\
\omega & =(4114.3813 \pm 11.8908) \mathrm{s}^{-1}, \quad \text { and } \\
\phi & =-0.0034 \pm 0.0082 .
\end{aligned}
$$

The envelope $b^{\text {osc }}(t)$ of the fast oscillations is consistent with the law

$$
b^{\mathrm{osc}}(t)=\sin \left[\xi_{\mathrm{EDM}}(d)\right] \cdot \cos (\omega t) .
$$

According to [18], the EDM induced angular velocity $\omega$ in Eq. (78) can be expressed through the EDM resonance strength $\varepsilon^{\mathrm{EDM}}$ and the orbital angular velocity frequency $\omega_{\text {rev }}$, via

$$
\omega=\varepsilon^{\mathrm{EDM}} \cdot \omega_{\mathrm{rev}} .
$$

Now we can apply Eq. (84) to interpret the result for the initial slope for $d=10^{-20} e \mathrm{~cm}$, given in Eq. (74). Equations (80) and (81) entail

$\left.\dot{p}_{y}(t)\right|_{t=0}=\cos \xi_{\mathrm{EDM}} \cdot\left|\varepsilon_{\mathrm{EDM}}\right| \cdot \omega_{\mathrm{rev}}=4.305033 \times 10^{-6}$,

where $\varepsilon_{\mathrm{EDM}}$ from Eq. (44) was used with $\xi_{\mathrm{EDM}}$ from Table I. Nice agreement is obtained with the value of the initial slope from the simulations [Eq. (74)].

In terms of the initial slope, the resonance strength is given by

$$
\varepsilon^{\mathrm{EDM}}=\frac{\left.\dot{p}_{y}(t)\right|_{t=0}}{a \cos \phi_{\mathrm{rf}}} \cdot \frac{1}{\omega_{\mathrm{rev}}} .
$$

While the slopes can be easily determined as a function of $\phi_{\mathrm{rf}}$, the latter method using Eq. (86) clearly also requires knowledge about the oscillation amplitude $a$. Knowing the initial slopes alone does not allow one to determine the resonance strength $\varepsilon^{\mathrm{EDM}}$.

Using the technique of variation of $\phi_{\mathrm{rf}}$, as shown in Fig. 11, Fig. 13 yields an initial slope of

$$
\left.\dot{p}_{y}(t)\right|_{t=0}=(3959 \pm 35) \mathrm{s}^{-1},
$$




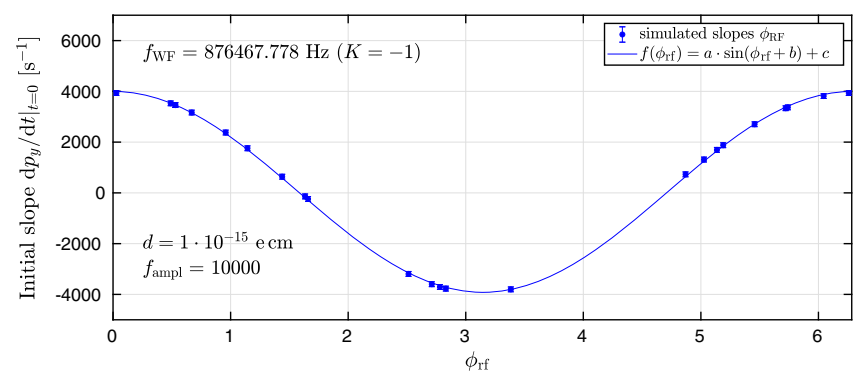

FIG. 13. Initial slope as a function of 24 random values of $\phi_{\mathrm{rf}}$ using a field amplification factor $f_{\mathrm{ampl}}=10^{4}$ and the indicated EDM. The simulated data are fitted using the function indicated in the inset. The resulting parameters are $a=(3959.122 \pm 35.344), \quad b=(4135.901 \pm 0.009), \quad$ and $c=(39.861 \pm 25.995)$. Each data point is obtained from a graph like the one shown in Fig. 9, but for ten turns and 1001 points.

which agrees numerically well within errors with the value given in the last line of Eq. (79).

\section{Determination of the running spin tune, based on the polarization evolution $\vec{S}_{2}(t)$}

The standard definition of the spin tune as a rotation around the local stable spin axis $\vec{n}_{s}$ at every point in the machine holds for a static machine. It does not involve a time dependence of the polarization evolution, like the one generated by the $\mathrm{rf}$ Wien filter. If the polarization is time dependent, the term running or instantaneous spin tune will be used in the following. In case there is a timedependent or instantaneous spin tune, the direction of $\vec{n}_{s}$ also changes as a function of time, i.e., $\vec{n}_{s} \equiv \vec{n}_{s}(t)$ (see further Sec. III E 5).

Using the numerical simulations for $\vec{S}_{2}(t)$, or any other spin-evolution function, one can numerically determine the running spin tune in the following way. For this one needs three spin vectors from the spin-evolution function, say

$$
\begin{aligned}
& \vec{a}=\vec{S}_{2}(t), \\
& \vec{b}=\vec{S}_{2}\left(t+T_{\mathrm{rev}}\right), \quad \text { and } \\
& \vec{c}=S_{2}\left(t+2 \cdot T_{\mathrm{rev}}\right) .
\end{aligned}
$$

Using these three vectors, two more vectors are constructed,

$$
\vec{d}(t)=\vec{a}-\vec{b} \quad \text { and } \quad \vec{e}(t)=\vec{a}-\vec{c} .
$$

The in-plane angle between $\vec{d}(t)$ and $\vec{e}(t)$ can be used to determine the running, time-dependent spin tune $\nu_{s}(t)$. To this end, we define the normal vector $\vec{N}$ of the plane that contains $\vec{d}$ and $\vec{e}$,

$$
\vec{N}=\frac{\vec{d} \times \vec{e}}{|\vec{d} \times \vec{e}|}
$$

which corresponds to the instantaneous spin axis. Using $\vec{N}$, we find the in-plane components of $\vec{b}$ and $\vec{c}$, via

$$
\vec{b}_{\perp}=\vec{b} \times \vec{N} \quad \text { and } \quad \vec{c}_{\perp}=\vec{c} \times \vec{N}
$$

The normalized versions of these vectors are called

$$
\vec{f}=\frac{\vec{b}_{\perp}}{\left|\vec{b}_{\perp}\right|} \quad \text { and } \quad \vec{g}=\frac{\vec{c}_{\perp}}{\left|\vec{c}_{\perp}\right|}
$$

and the running spin tune is determined from

$$
\nu_{s}(t)=\frac{1}{2 \pi} \frac{G}{|G|} \arctan \left|\frac{\vec{f}(t) \times \vec{g}(t)}{\vec{f}(t) \cdot \vec{g}(t)}\right| .
$$

The factors in front of arctangent take care that $\nu_{s}(t)$ generates the correct sign based on the $G$-factor and the number of spin precessions per turn.

As a cross-check of the algorithm, with the rf Wien filter switched off, for the beam conditions given in Table I, Eq. (93) yields

for $d=0: \nu_{s}^{(0)}=G \gamma=-1.609771846321990 \times 10^{-1}$,

$$
\text { for } \begin{aligned}
d & =1 \times 10^{-20} e \mathrm{~cm}: \nu_{s}^{(1)}=\frac{G \gamma}{\cos \xi_{\mathrm{EDM}}} \\
& =-1.609771846329495 \times 10^{-1}, \quad \text { and } \\
\Delta \nu_{s} & =\nu_{s}^{(0)}-\nu_{s}^{(1)}=+7.505 \times 10^{-13},
\end{aligned}
$$

where all three numbers have been calculated using Eq. (93). As an additional cross-check, the difference of the spin tunes yields

$$
\frac{\nu_{s}^{(0)}}{\cos \xi_{\mathrm{EDM}}}-\nu_{s}^{(1)} \approx 10^{-16},
$$

which is very close to the achievable machine precision (see footnote 8).

During a revolution in the machine, as prescribed by $\vec{S}_{2}(t)$ using Eq. (42), the spin tune remains constant during each turn [see Fig. 14(a)]. When the rf Wien filter is switched on, due to the additional spin rotation in the timevarying rf field, the instantaneous spin tune jumps from turn to turn. As depicted in Fig. 14(b), the oscillation amplitude of the spin tune variation due to the rf Wien filter using a power of $1 \mathrm{~kW}$ (see Table I) is well consistent with the expectation from the spin rotation formalism: 


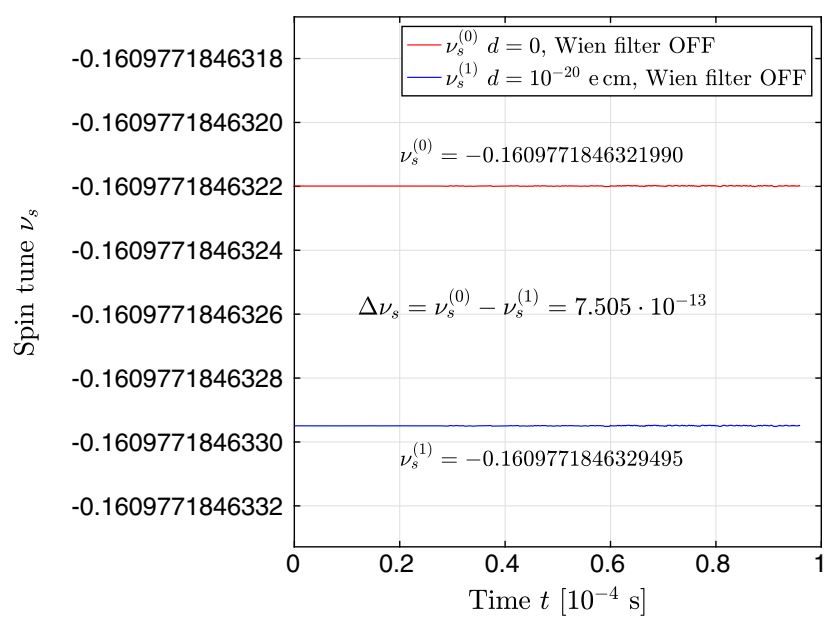

(a)

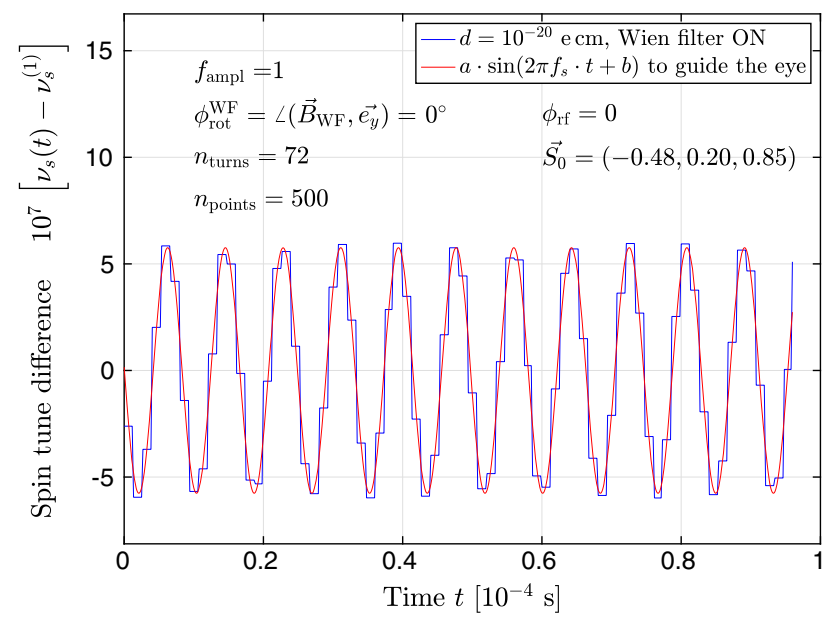

(b)

FIG. 14. The graph in panel (a) shows the spin tune in the machine, calculated using Eq. (93) for the conditions listed in Table I for $d=0$ (red) and $d \neq 0$ (blue) and rf Wien filter switched OFF. Panel (b) shows the time dependence when the rf Wien filter is switched $\mathrm{ON}$ in EDM mode with $f_{\mathrm{ampl}}=1$. The red curve indicates the spin oscillation frequency $f_{s}$ from Eq. (17), and the blue line denotes the running spin tune difference $\nu_{s}(t)-\nu_{s}^{(1)}$ for each turn. It should be noted that the initial spin vector $\vec{S}_{0}$ is not in the ring $(x z)$ plane (see Fig. 1).

$$
a=(5.7 \pm 0.2) \times 10^{-7} \approx \frac{\left|\psi_{\mathrm{WF}}\right|}{2 \pi}=6.0 \times 10^{-7} .
$$

The average spin tune, however, remains constant.

\section{Instantaneous spin orbit determination based on $\vec{S}_{2}(t)$}

The running spin orbit vector $\vec{n}_{s}$ can be easily determined from the procedure described in the previous section, using the normal vector $\vec{N}$, defined in Eq. (90),

$$
\vec{n}_{s}(t)=\vec{N}(t)
$$

Similarly to the running (instantaneous) spin tune, the instantaneous spin orbit exhibits oscillating in-plane polarization components.

\section{POLARIZATION EVOLUTION WITH rf WIEN FILTER AND SOLENOIDS}

\section{A. Evolution equation with additional static solenoids}

In the course of this paper, with the rf Wien filter in EDM mode $\left(\vec{B}^{\mathrm{WF}} \| \vec{e}_{y}\right)$, the EDM interaction with the motional electric field in the ring was the only source of up-down spin oscillations.

In the following, two static solenoids in the straight sections will be added to the ring. Besides that, we shall make an allowance for rotations of the rf Wien filter around the longitudinal $\vec{e}_{z}$ (momentum) direction. Such rotations induce a radial magnetic rf field, and, in conjunction with the solenoidal magnetic fields, we start mixing the EDM and MDM induced rotations. The idea, common to all EDM experiments, is to disentangle the EDM signal using an extrapolation to a vanishing MDM contribution $[25,26]$.

With two static solenoids added to the ring, the resulting sequence of elements is depicted in Fig. 15. The one-turn ring matrix can be split into two arcs, one arc made of the dipole magnets $D_{1}$ to $D_{12}$, and the second arc made of dipoles $\mathrm{D}_{13}$ to $\mathrm{D}_{24}$. Since

$\mathbf{U}_{\text {ring }}\left(\vec{c}, T_{\text {rev }}\right)=\mathbf{U}_{\text {ring }}^{\operatorname{arc} 2}\left(\vec{c}, T_{\text {rev }} / 2\right) \times \mathbf{U}_{\text {ring }}^{\operatorname{arc} 1}\left(\vec{c}, T_{\text {rev }} / 2\right)$,

the two additional solenoids can be inserted before and behind arc 2, leading to

$$
\begin{aligned}
\mathbf{U}_{\text {ring }}^{2 \text { sol }} & \left(\vec{c}, T_{\text {rev }}, \chi_{\text {rot }}^{\mathrm{S}_{1}}, \chi_{\text {rot }}^{\mathrm{S}_{2}}\right) \\
= & \mathbf{R}\left(\vec{e}_{z}, \chi_{\text {rot }}^{\mathrm{S}_{2}}\right) \times \mathbf{U}_{\text {ring }}^{\operatorname{arc} 2}\left(\vec{c}, T_{\text {rev }} / 2\right) \times \mathbf{R}\left(\vec{e}_{z}, \chi_{\text {rot }}^{\mathrm{S}_{1}}\right) \\
& \times \mathbf{U}_{\text {ring }}^{\operatorname{arc} 1}\left(\vec{c}, T_{\text {rev }} / 2\right),
\end{aligned}
$$

invoking again the generic rotation matrix $\mathbf{R}\left(\vec{e}_{z}, \chi_{\text {rot }}\right)$ from Eq. (12).

In a similar fashion as in Eq. (42), one can then write for the polarization evolution, 


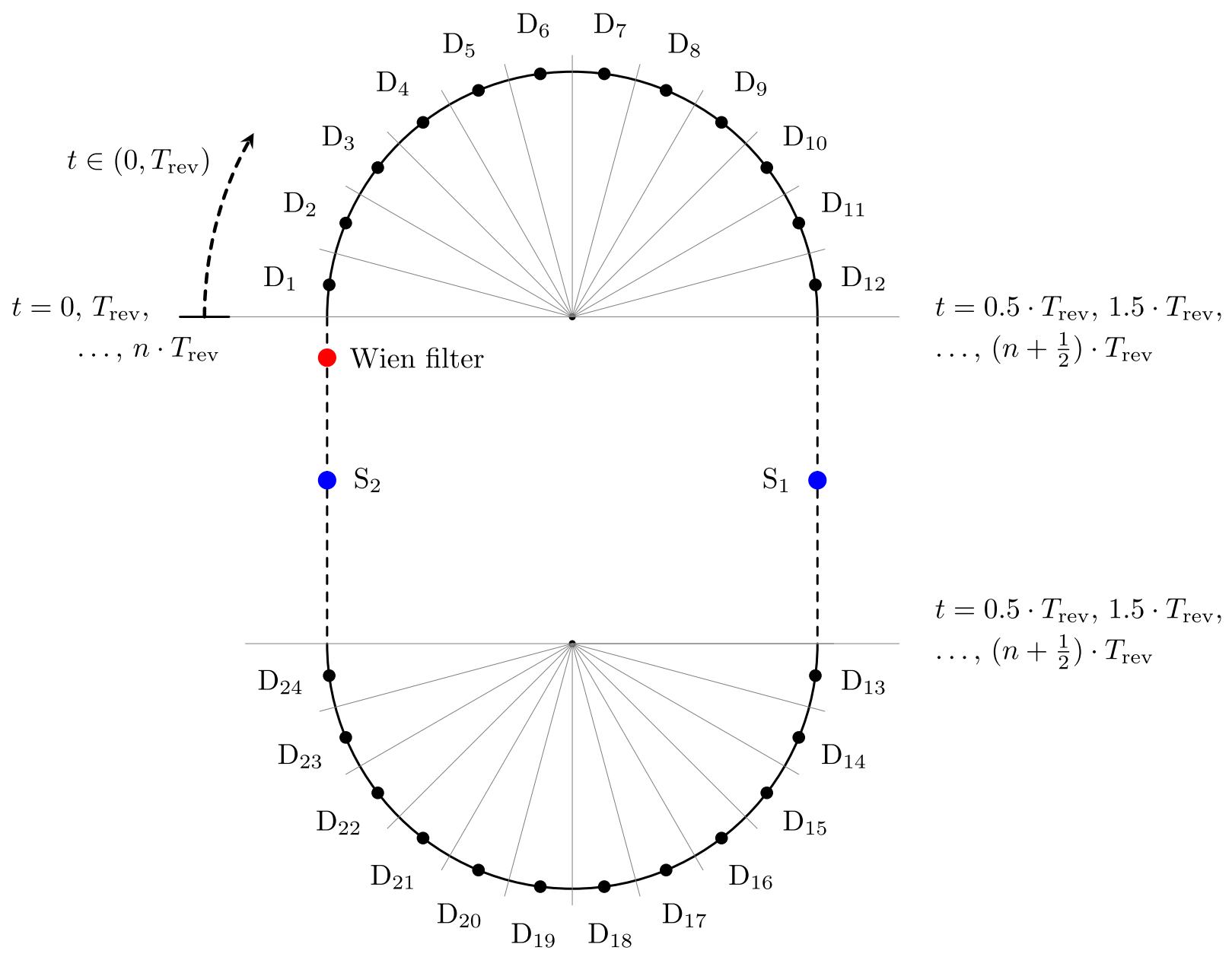

FIG. 15. Sequence of elements in the ring, corresponding to Eq. (100), including besides the rf Wien filter, also two static solenoids $\mathrm{S}_{1}$ and $\mathrm{S}_{2}$.

$$
\begin{aligned}
\vec{S}_{3}(t)= & \underbrace{\mathbf{U}_{\text {ring }}\left(\vec{c}, t-n \cdot T_{\text {rev }}\right)}_{\text {rest of last turn }} \times \underbrace{\left[\mathbf{U}_{\mathrm{WF}}\left(t=n \cdot T_{\text {rev }}\right) \times \mathbf{U}_{\text {ring }}^{2 \text { sol }}\left(\vec{c}, T_{\text {rev }}, \chi_{\text {rot }}^{\mathrm{S}_{1}}, \chi_{\text {rot }}^{\mathrm{S}_{2}}\right)\right]}_{\text {turn n }} \times \cdots \\
& \times \underbrace{\left[\mathbf{U}_{\mathrm{WF}}\left(t=2 \cdot T_{\text {rev }}\right) \times \mathbf{U}_{\text {ring }}^{2 \text { sol}}\left(\vec{c}, T_{\text {rev }}, \chi_{\text {rot }}^{\mathrm{S}_{1}}, \chi_{\text {rot }}^{\mathrm{S}_{2}}\right)\right]}_{\text {turn } 2} \times \underbrace{\left[\mathbf{U}_{\mathrm{WF}}\left(t=T_{\text {rev }}\right) \times \mathbf{U}_{\text {ring }}^{2 \text { sol }}\left(\vec{c}, T_{\text {rev }}, \chi_{\text {rot }}^{\mathrm{S}_{1}}, \chi_{\text {rot }}^{\mathrm{S}_{2}}\right)\right]}_{\text {turn } 1} \times \vec{S}_{0} .
\end{aligned}
$$

\section{B. Spin-rotation angle in a static solenoid}

In a solenoidal magnet with a field integral $\mathrm{BDL}=$ $\int B_{\|} \mathrm{d} \ell$, the spins are rotated around the longitudinal direction $\vec{e}_{z}$, and the rotation angle is given by

$$
\chi_{\mathrm{rot}}^{\mathrm{Sol}}=-\frac{q}{m} \cdot \frac{(1+G)}{\gamma \beta c} \int B_{\|} \mathrm{d} \ell .
$$

The spin-rotation angle in the solenoid for deuterons at a momentum of $P=970 \mathrm{MeV} / c$, normalized to the magnetic field integral, amounts to

$$
\frac{\chi_{\mathrm{rot}}^{\mathrm{Sol}}}{\int B_{\|} \mathrm{d} \ell}=-0.264872 \operatorname{rad~T}^{-1} \mathrm{~m}^{-1}
$$

\section{Spin tune and spin closed orbit with solenoids using $\vec{S}_{\mathbf{3}}(t)$}

In the following, the abbreviation, e.g., $\chi_{\text {rot }}^{\text {Sol } 1}=\chi_{1}$ is used. For an ideal ring, free of magnetic imperfections, the spin tune change $\Delta \nu_{s}\left(\chi_{1}, \chi_{2}\right)$, due to solenoids $S_{1}$ and $S_{2}$ in the ring (see Fig. 15), the left side of Eq. (30) of Ref. [18] can be approximated by $\pi \Delta \nu_{s}\left(\chi_{1}, \chi_{2}\right) \cdot \sin \left(\pi \nu_{s}^{0}\right)$, where $\nu_{s}^{0}$ denotes the unperturbed spin tune in the machine. For small spin-rotation angles in the solenoids, Eq. (30) of [18] can thus be approximated by

$$
\Delta \nu_{s}\left(\chi_{1}, \chi_{2}\right)=\frac{2 \chi_{1} \chi_{2}+\cos \left(\pi \nu_{s}^{0}\right) \cdot\left(\chi_{1}^{2}+\chi_{2}^{2}\right)}{8 \pi \sin \left(\pi \nu_{s}^{0}\right)}
$$



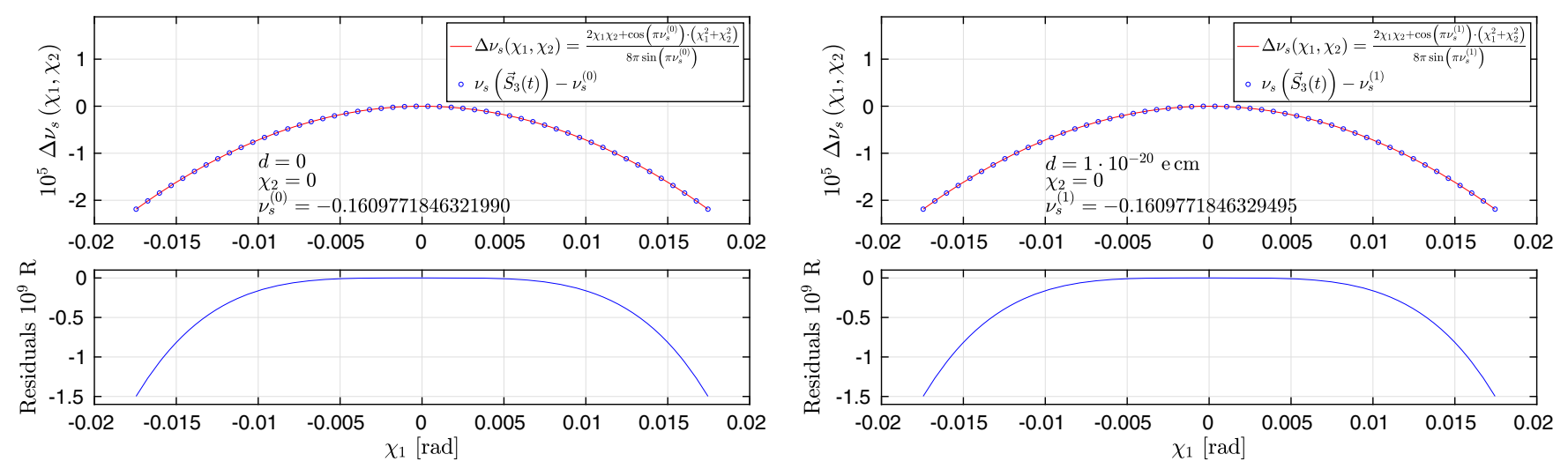

(a)

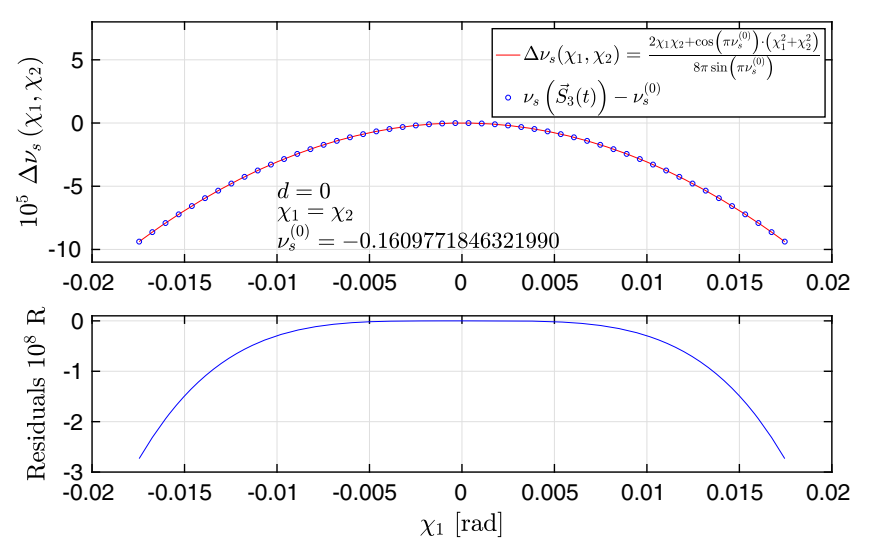

(c) (b)
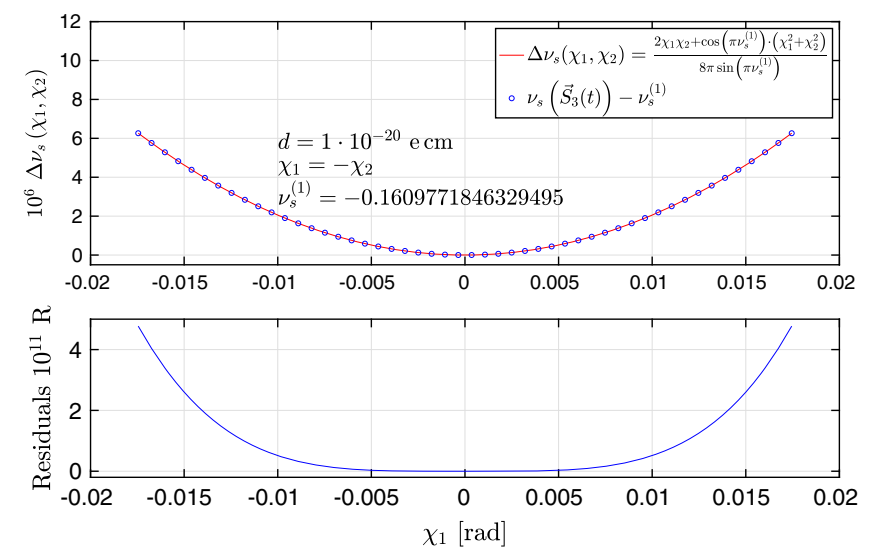

(d)

FIG. 16. Change of the spin tune $\Delta \nu_{s}\left(\chi_{1}, \chi_{2}\right)$ for deuterons using two solenoids in the machine (as indicated in Fig. 15) under the conditions of Table I using Eq. (93) and $\vec{S}_{3}(t)$ from Eq. (100). Panels (a) and (c) show for $d=0 \Delta \nu_{s}\left(\chi_{1}, \chi_{2}\right)=\nu_{s}(t)-\nu_{s}^{(0)}$, while (b) and (d) show for $d=10^{-20} e \mathrm{~cm} \Delta \nu_{s}\left(\chi_{1}, \chi_{2}\right)=\nu_{s}(t)-\nu_{s}^{(1)}$. Panels (a) and (b) $\chi_{2}=0$; (c) $\chi_{1}=\chi_{2}$ : and (d) $\chi_{1}=-\chi_{2}$. $\nu_{s}^{(0)}$ and $\nu_{s}^{(1)}$ are given in the insets [see also Eq. (94)]. The residuals $R$ show the difference between the simulations and the approximations using Eq. (103).

In order to validate the spin-evolution equation for $\vec{S}_{3}(t)$, given in Eq. (100), in Fig. 16 the spin-tune changes $\Delta \nu_{s}$ are compared to the approximation of Eq. (103) for four different cases.

\section{Spin-closed orbit in a nonideal lattice}

The static solenoids or magnetic imperfections in the ring affect the spin-closed orbit vector $\vec{n}_{s}=\vec{c}$ in the machine. The situation is similar to the one depicted in Fig. 1, but there, only the tilt due to the EDM was taken into account. The presence of static solenoids in the ring can be numerically evaluated using Eq. (97) with $\vec{S}_{3}(t)$ from Eq. (100).

Since the time $t$ begins to count right behind the rf Wien filter (see Fig. 15), evaluation of Eq. (97) at $t=T_{\text {rev }}$ \{or integer multiples of $T_{\text {rev }}$ [see Eq. (40)]\}, yields the orientation of the spin-closed orbit vector $\vec{c}$ at the $\mathrm{rf}$ Wien filter

$$
\vec{c}=\vec{n}_{s}\left(t=T_{\text {rev }}\right) .
$$

Figure 17 shows how the stable spin axis $\vec{c}=\left(c_{x}, c_{y}, c_{z}\right)$ at the rf Wien filter is affected by the two solenoids $S_{1}$ and $\mathrm{S}_{2}$, and the presence of an EDM $d$. For comparisons, a number of special cases was numerically evaluated and is listed in Table V.

\section{E. Strength of the EDM resonance}

As depicted in Fig. 14, and already discussed in Sec. III E 4, the operation of the rf Wien filter modulates the spin tune. While the average spin tune is equal to the one obtained when the rf Wien filter is switched off, solenoids and magnet misalignments in the ring, however, affect the spin tune. Therefore, the spin-precession frequency and thus the frequency at which the rf Wien filter should be operated, differs from the unperturbed spin tune. The spin tune $\nu_{s}$ must be determined anew for every 


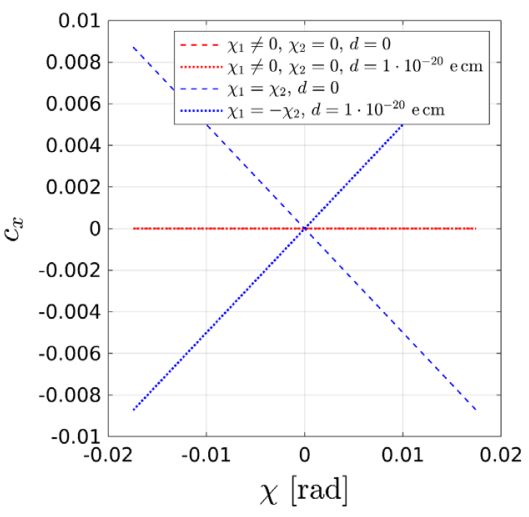

(a)

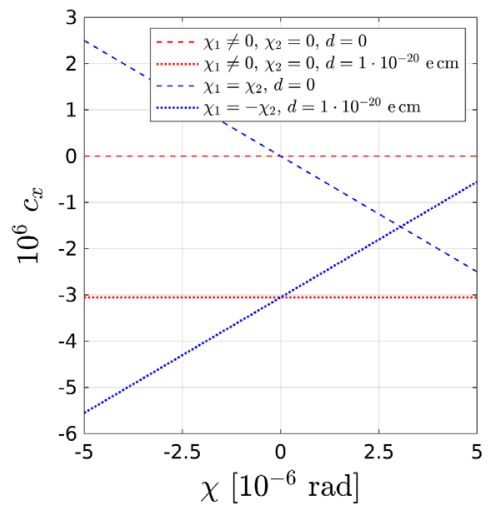

(d)

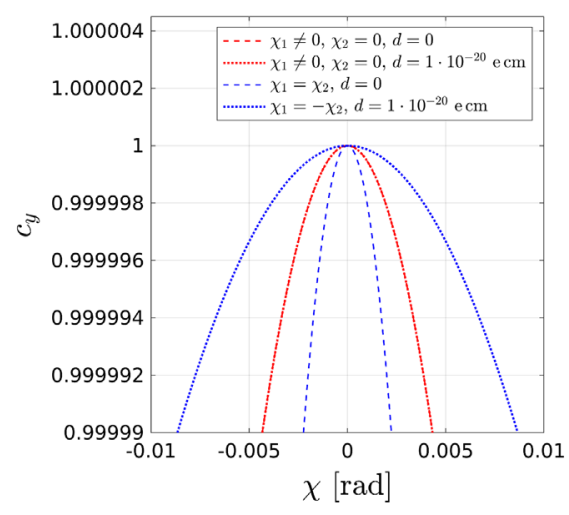

(b)

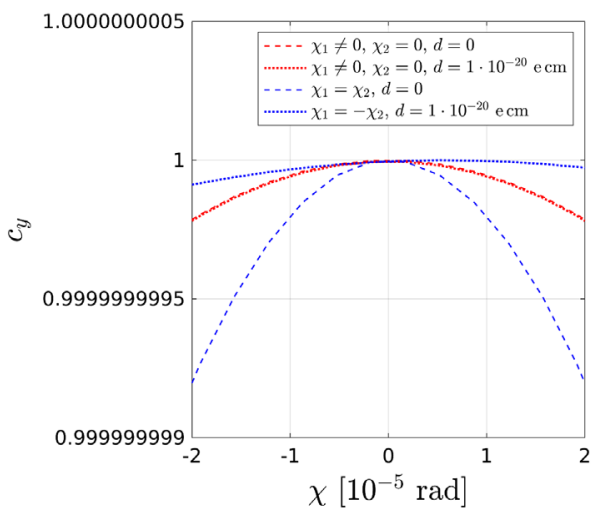

(e)

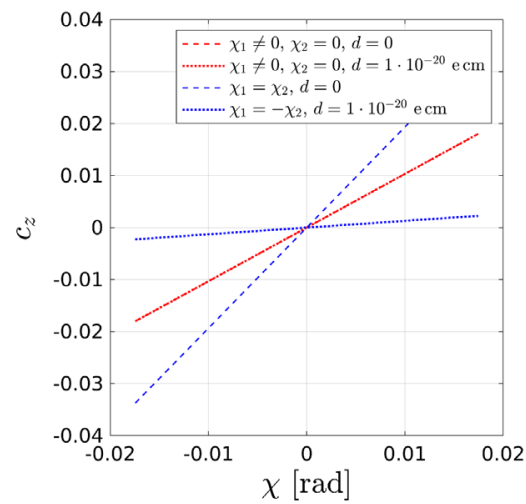

(c)

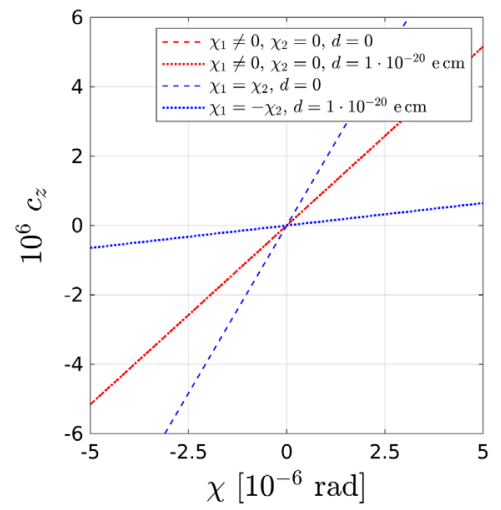

(f)

FIG. 17. Six panels showing the components of $\vec{c}=\left(c_{x}, c_{y}, c_{z}\right)$ at the rf Wien filter for different combinations of spin rotations in the two solenoids in the ring (see Fig. 15), for deuterons at a momentum of $P=970 \mathrm{MeV} / c$.

solenoid setting to ensure that the resonance frequency for the rf Wien filter is given by

$$
f_{\mathrm{WF}}=\left(K+\nu_{s}\right) \cdot f_{\text {rev }}, \quad K \in \mathbb{Z},
$$

and this frequency needs to be used in $\psi(t))$ [Eq. (35)], as it controls the rf Wien filter spin-rotation matrix $\mathbf{R}\left[\vec{n}_{\mathrm{WF}}, \psi(t)\right]$ [Eq. (38)].

The EDM resonance strength $\varepsilon^{\mathrm{EDM}}$, actually a resonance tune, is defined as the ratio of the angular velocity of the vertical polarization oscillation $\Omega^{p_{y}}$ induced by the EDM relative to the orbital angular velocity $\Omega^{\text {rev }}$,

$$
\varepsilon^{\mathrm{EDM}}=\frac{\Omega^{p_{y}}}{\Omega^{\mathrm{rev}}}
$$

Since $\Omega_{p_{y}}$ corresponds to $\omega$ [first line in Eq. (79)], the resonance strength can in principle be determined from the sole observation of $\Omega_{p_{y}}$.

Alternatively, the resonance strength can be determined from the last line in Eq. (79) via

$$
\varepsilon^{\mathrm{EDM}}=\frac{\left.\dot{p}_{y}(t)\right|_{t=0}}{a \cos \phi} \cdot \frac{1}{\Omega^{\mathrm{rev}}},
$$

TABLE V. Components of the spin closed orbit vector $\vec{c}=\left(c_{x}, c_{y}, c_{z}\right)$ at the rf Wien filter, for different settings of the solenoids $\mathrm{S}_{1}$ and $\mathrm{S}_{2}$ in the machine (see Fig. 15).

\begin{tabular}{lccccc}
\hline \hline$\chi_{1}[\mathrm{deg}]$ & $\chi_{2}[\mathrm{deg}]$ & $d[e \mathrm{~cm}]$ & $c_{x}$ & $c_{y}$ & $c_{z}$ \\
\hline 0 & 0 & 0 & 0.000000 & 1.000000 & 0.000000 \\
0 & 0 & $10^{-20}$ & $-3.053662 \times 10^{-6}$ & 1.000000 & $4.255557 \times 10^{-17}$ \\
1 & 0 & $10^{-20}$ & $-3.053167 \times 10^{-6}$ & $9.998378 \times 10^{-1}$ & $1.801136 \times 10^{-2}$ \\
0 & 1 & $10^{-20}$ & $-8.728505 \times 10^{-3}$ & $9.998378 \times 10^{-1}$ & $1.575676 \times 10^{-2}$ \\
1 & 1 & $10^{-20}$ & $-8.724615 \times 10^{-3}$ & $9.993921 \times 10^{-1}$ & $3.375307 \times 10^{-2}$ \\
1 & -1 & $10^{-20}$ & $8.723460 \times 10^{-3}$ & $9.999594 \times 10^{-1}$ & $2.254871 \times 10^{-3}$ \\
-1 & 1 & $10^{-20}$ & $-8.729567 \times 10^{-3}$ & $9.999594 \times 10^{-1}$ & $-2.254871 \times 10^{-3}$ \\
-1 & -1 & $10^{-20}$ & $8.718511 \times 10^{-3}$ & $9.993922 \times 10^{-1}$ & $-3.375307 \times 10^{-2}$ \\
\hline \hline
\end{tabular}




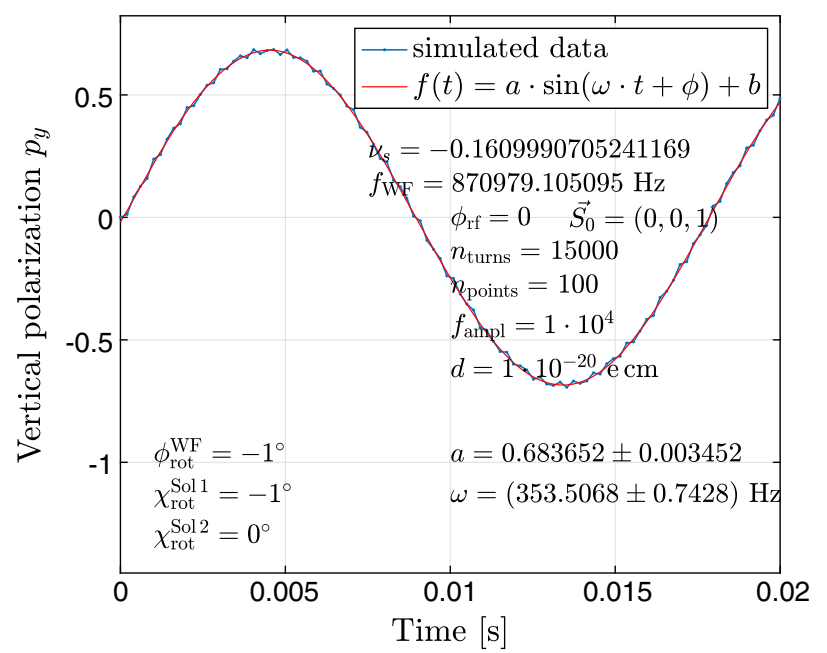

(a)

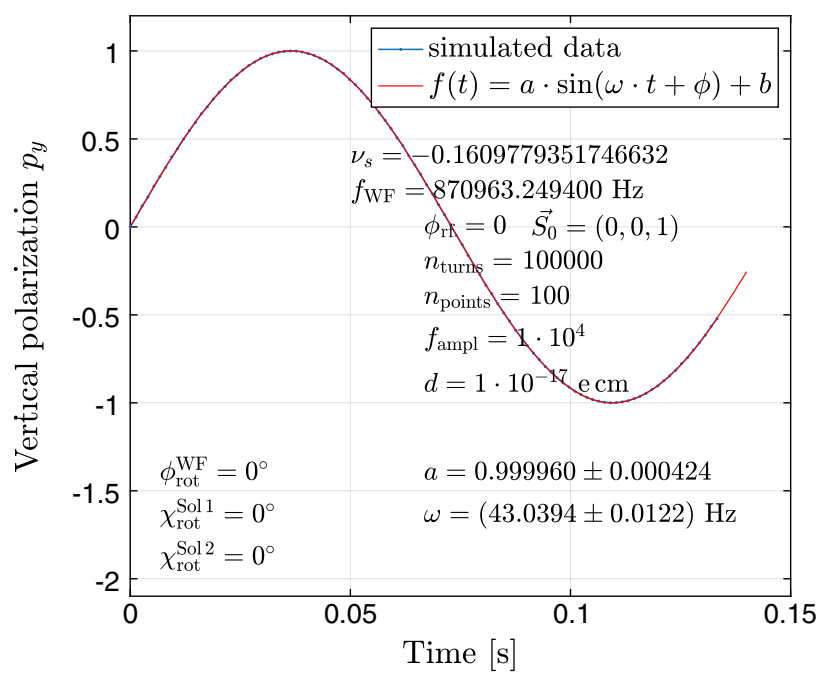

(b)

FIG. 18. Two examples for the evolution of $p_{y}(t)$ using $\vec{S}_{3}(t)$ from Eq. (100) for different combinations of Wien filter and solenoid spin rotation angle, denoted by $\left(\phi_{\mathrm{rot}}^{\mathrm{WF}}, \chi_{\mathrm{rot}}^{\mathrm{Sol} 1}\right)$, where $\chi_{\mathrm{rot}}^{\mathrm{Sol} 2}=0$. The parameters used for the calculation are indicated in each panel. For the beam, the conditions of Table I apply. The Wien filter is operated at harmonic $K=-1$. The EDM assumed in panel (b) is 1000 times larger than in (a). The ratio of the fitted oscillation amplitudes in panels (a) and (b) is compatible with the expectation of a factor $\sqrt{2} / 2$ [see Eq. (109)].

but this requires that the initial slopes need to be determined as a function of, e.g., $\phi=\phi_{\mathrm{rf}}$. The statistical aspects of this will be further elucidated in Sec. IV E 2.

\section{Evolution of $p_{y}(t)$ as a function of $\phi_{\mathrm{rot}}^{\mathrm{WF}}$ and $\chi_{\mathrm{rot}}^{\mathrm{Sol} 1}$}

The EDM resonance strength $\varepsilon^{\mathrm{EDM}}$ [Eq. (106)] manifests itself in the oscillation frequency, as illustrated in Fig. 18 for two pairs of Wien filter rotation angle and spin-rotation angle in the solenoid $S_{1},\left(\phi_{\mathrm{rot}}^{\mathrm{WF}}, \chi_{\mathrm{rot}}^{\mathrm{Sol} 1}\right)$, while $\chi_{\mathrm{rot}}^{\mathrm{Sol} 2}=0$.

The resulting oscillation pattern of $p_{y}$ is fitted using

$$
f(t)=a \sin (\omega t+\phi)+b,
$$

amplitude $a$ and frequency $\omega$ are given in each panel, together with various other parameters. The calculation for the ideal ring situation in panel (b) uses a 1000 times larger assumed EDM value of $d=10^{-17} e \mathrm{~cm}$ and a larger number of turns $n_{\text {turns }}=100000$, in order to make the oscillations of $p_{y}(t)$ visible when the rf Wien filter does not contribute a sideways magnetic field.

\section{Comparison of $\varepsilon^{\mathrm{EDM}}$ from $\Omega^{p_{y}}$ and $\left.\dot{p}_{y}(t)\right|_{t=0}$ by variation of $\phi_{\mathrm{rf}}$}

One would expect that the variation of the rf phase $\phi_{\mathrm{rf}}$ will affect the resulting oscillation amplitudes $a$ and offsets $b$ of Fig. 18, while the oscillation frequencies $\omega$, and thus the resonance strengths $\varepsilon^{\mathrm{EDM}}$, remain unchanged.

In the panels of Fig. 19, for the same combinations of $\left(\phi_{\mathrm{rot}}^{\mathrm{WF}}, \chi_{\mathrm{rot}}^{\mathrm{Sol} 1}\right)$, shown in Fig. $18,\left.\dot{p}_{y}(t)\right|_{t=0}$ and the oscillation frequency $\omega$ are computed for 36 randomly picked values of $\phi_{\mathrm{rf}}$. The graph illustrates that in the presence of solenoid fields and $\mathrm{rf}$ Wien filter rotations, the determination of $\left.\dot{p}_{y}(t)\right|_{t=0}$ by variation of $\phi_{\text {rf }}$, making use of Eq. (107) yields results comparable to the direct determination of the resonance strength from the oscillation frequency $\Omega^{p_{y}}$ via Eq. (106). The oscillation amplitudes $a$ and $\left.\dot{p}_{y}\right|_{t=0}$ exhibit an identical dependence on $\phi_{\mathrm{rf}}$, while the obtained resonance tune $\varepsilon^{\mathrm{EDM}}$ remains constant over the whole range of $\phi_{\mathrm{rf}}$.

The resonance strengths extracted from $\left.\dot{p}_{y}(t)\right|_{t=0}$ and $\Omega^{p_{y}}$ make use of the very same simulated data. The results are summarized in Table VI, where for the numbers that should match, the markers (A), (B), and (C) are used. Although the different extraction methods show good overall agreement, the uncertainties of $\varepsilon^{\mathrm{EDM}}\left(\Omega^{p_{y}}\right)$, however, are substantially smaller than those from $\varepsilon^{\mathrm{EDM}}\left(\left.\dot{p}_{y}\right|_{t=0}\right)$ by a factor of at least 20. The reason for this is that in general frequencies can be measured more accurately than other quantities, and the determination of $\varepsilon^{\operatorname{EDM}}\left(\Omega^{p_{y}}\right)$ involves fewer uncertainties in the error propagation. The most accurate determinations are obtained from $\Omega^{p_{y}}$ when $\chi_{\text {rot }}^{\text {Sol } 1}=0$.

In the following, we briefly comment on some features of the results obtained so far (Fig. 18, Table VI). We observe that numerically $2 \sin \pi \nu_{s}=1.0041 \simeq 1$. Then, according to the Appendix, we expect

$$
a\left(-1^{\circ},-1^{\circ}\right)=\cos \left(\frac{\pi}{4}\right) \cdot a\left(0^{\circ}, 0^{\circ}\right),
$$

in good agreement with the results shown in Fig. 19. The resonance tunes determined from $\left.\dot{p}_{y}\right|_{t=0}$ and from $\Omega^{p_{y}}$ are 

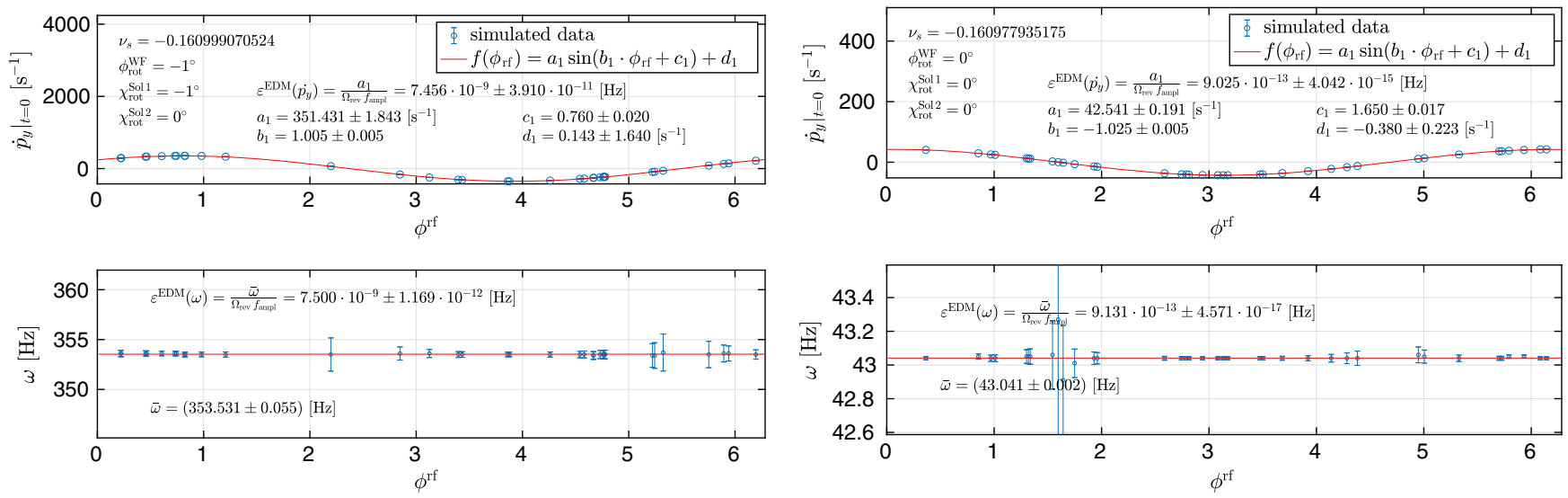

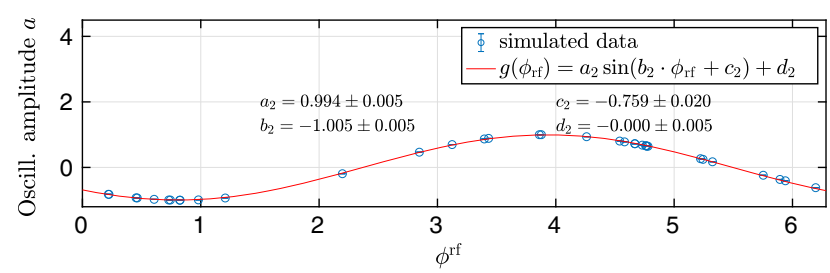

(a)

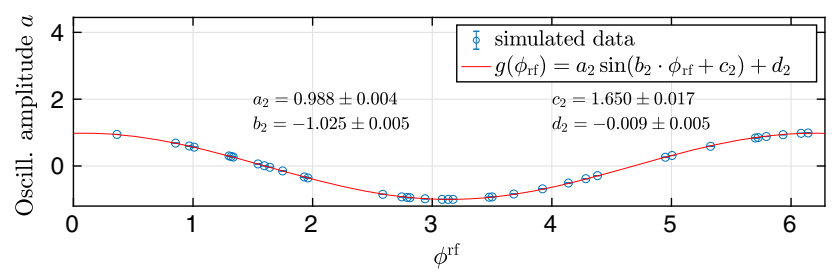

(b)

FIG. 19. Two examples showing 36 random values of $\phi_{\mathrm{rf}}$ that are used to obtain the resonance strengths $\varepsilon^{\mathrm{EDM}}$ from graphs like the ones shown in Fig. 18, using Eqs. (106) and (107) for combinations of the Wien filter and solenoid spin rotation angle, denoted by $\left(\phi_{\mathrm{rot}}^{\mathrm{WF}}, \chi_{\mathrm{rot}}^{\mathrm{Sol}}\right)$. Depicted here as a function of the randomly chosen $\phi_{\mathrm{rf}}$ are the extracted initial slopes $\left.\dot{p}_{y}(t)\right|_{t=0}, \omega=\Omega^{p_{y}}$, and the amplitude $a$ of the $p_{y}$ oscillation [Eq. (108)]. The parameters used for the calculation are $n_{\text {turns }}=2 \times 10^{4}, n_{\text {points }}=200$, and $d=10^{-20} e \mathrm{~cm}$. To enhance the effect, in panel (b), $n_{\text {turns }}=10^{5}$, and the assumed EDM is $d=10^{-17} e \mathrm{~cm}$, i.e., 1000 times larger than in (a). For the beam, the conditions of Table I apply. The rf Wien filter is operated at harmonic $K=-1$. The extracted resonance strengths are summarized in Table VI.

identical. For the above reason of $2 \sin \pi \nu_{s} \simeq 1$ and small EDM contributions, the following equalities hold:

$$
\begin{aligned}
& \varepsilon^{\mathrm{EDM}}\left(-1^{\circ},-1^{\circ}\right)=\varepsilon^{\mathrm{EDM}}\left(1^{\circ}, 1^{\circ}\right), \quad \text { and } \\
& \varepsilon^{\mathrm{EDM}}\left( \pm 1^{\circ},-1^{\circ}\right)=\sqrt{2} \cdot \varepsilon^{\mathrm{EDM}}\left(-1^{\circ}, 0^{\circ}\right) .
\end{aligned}
$$

\section{F. Resonance strength $\varepsilon^{\mathrm{EDM}}$ for random points $\left(\phi_{\mathrm{rot}}^{\mathrm{WF}}, \chi_{\mathrm{rot}}^{\mathrm{Sol} 1}\right)$}

The resonance strengths shown in Fig. 20 are obtained using the fit function of Eq. (108) $\left(\omega=\Omega^{p_{y}}\right)$ and then Eq. (106) for a set of randomly chosen pairs of $\left(\phi_{\mathrm{rot}}^{\mathrm{WF}}, \chi_{\mathrm{rot}}^{\mathrm{Sol} 1}\right)$ and $\chi_{\text {rot }}^{\text {Sol } 2}=0$. For all points, $\phi_{\text {rf }}=0$ and $\vec{S}_{0}=(0,0,1)$,

TABLE VI. Resonance strengths extracted from Fig. 19 for nine different combinations $\left(\phi_{\mathrm{rot}}^{\mathrm{WF}}, \chi_{\mathrm{rot}}^{\mathrm{Sol}} 1\right)$ for an otherwise ideal COSY ring assuming a deuteron EDM of $d=10^{-20} e \mathrm{~cm}$ [for (b), at $\left(0^{\circ}, 0^{\circ}\right), d=10^{-17} e \mathrm{~cm}$ ]. The beam conditions are given in Table I using the real field magnitudes of the rf Wien filter, since $f_{\text {ampl }}$ has been divided out. For the calculations $n_{\text {turns }}=2 \times 10^{4}$ and $n_{\text {points }}=200$, except for (b), where $n_{\text {turns }}=10^{5}$. According to the equalities of Eq. (110), within the errors, the sets of numbers with markers (A), (B),

\begin{tabular}{|c|c|c|c|c|}
\hline \multicolumn{2}{|l|}{$\left[10^{-11} \mathrm{~Hz}\right]$} & \multicolumn{3}{|c|}{$\left(\phi_{\mathrm{rot}}^{\mathrm{WF}}, \chi_{\mathrm{rot}}^{\mathrm{Sol} 1}\right)$} \\
\hline$\varepsilon^{\mathrm{EDM}}$ & $\begin{array}{l}\text { From }\left.\dot{p}_{y}\right|_{t=0} \\
\text { From } \Omega^{p_{y}}\end{array}$ & $\begin{array}{l}\left(-1^{\circ},-1^{\circ}\right) \\
\text { (A) } 745.563 \pm 3.910 \\
\text { (A) } 750.017 \pm 0.117\end{array}$ & $\begin{array}{l}\left(0^{\circ},-1^{\circ}\right) \\
\text { (B) } 539.778 \pm 1.695 \\
\text { (B) } 538.659 \pm 0.099\end{array}$ & $\begin{array}{c}\left(1^{\circ},-1^{\circ}\right) \\
\text { (A) } 750.455 \pm 3.312 \\
\text { (A) } 749.840 \pm 0.128\end{array}$ \\
\hline$\varepsilon^{\mathrm{EDM}}$ & $\begin{array}{l}\text { From }\left.\dot{p}_{y}\right|_{t=0} \\
\text { From } \Omega^{p_{y}}\end{array}$ & $\begin{array}{l}\left(-1^{\circ}, 0^{\circ}\right) \\
\text { (C) } 517.167 \pm 2.741 \\
\text { (C) } 521.890 \pm 0.001\end{array}$ & $\begin{array}{c}\left(0^{\circ}, 0^{\circ}\right) \\
(90.251 \pm 0.404) \times 10^{-3} \\
(91.312 \pm 0.005) \times 10^{-3}\end{array}$ & $\begin{array}{c}\left(1^{\circ}, 0^{\circ}\right) \\
\text { (C) } 518.440 \pm 2.284 \\
\text { (C) } 521.681 \pm 0.001\end{array}$ \\
\hline$\varepsilon^{\mathrm{EDM}}$ & $\begin{array}{l}\text { From }\left.\dot{p}_{y}\right|_{t=0} \\
\text { From } \Omega^{p_{y}}\end{array}$ & $\begin{array}{c}\left(-1^{\circ}, 1^{\circ}\right) \\
\text { (A) } 748.511 \pm 3.249 \\
\text { (A) } 749.960 \pm 0.121\end{array}$ & $\begin{array}{c}\left(0^{\circ}, 1^{\circ}\right) \\
\text { (B) } 540.799 \pm 3.136 \\
\text { (B) } 538.619 \pm 0.129\end{array}$ & $\begin{array}{c}\left(1^{\circ}, 1^{\circ}\right) \\
\text { (A) } 749.413 \pm 3.891 \\
\text { (A) } 749.842 \pm 0.113\end{array}$ \\
\hline
\end{tabular}
and (C) should match. 


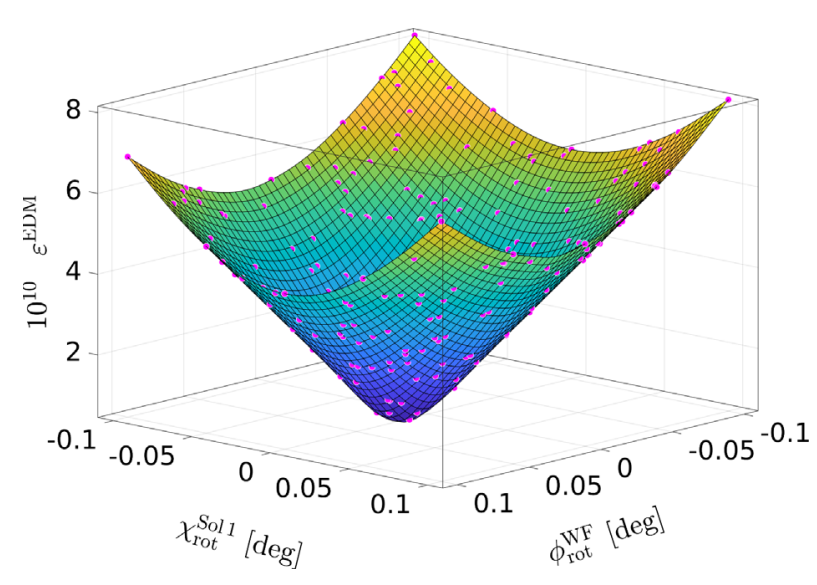

(a)

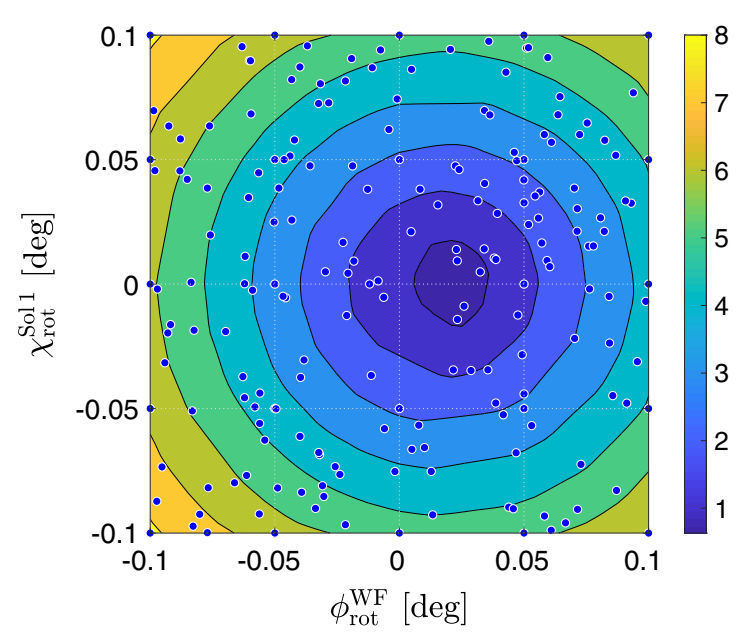

(b)

FIG. 20. Panels (a) and (b) show the resonance strengths $\varepsilon^{\mathrm{EDM}}$ as defined in Eq. (106) on a grid in the range $\phi_{\mathrm{rot}}^{\mathrm{WF}}=\left[-0.1^{\circ}, \ldots,+0 . n 1^{\circ}\right]$ and $\chi_{\mathrm{rot}}^{\mathrm{Sol} 1}=\left[-0.1^{\circ}, \ldots,+0.1^{\circ}\right]$ with an assumed EDM of $d=10^{-18} e \mathrm{~cm}$. Each point in panels (a) and (b) is obtained from a calculation with $n_{\text {turns }}=200000$ and $n_{\text {points }}=100$.

and an assumed EDM of $10^{-18} e \mathrm{~cm}$, for which the EDM tilt angle is $\xi_{\mathrm{EDM}} \approx 300 \mu \mathrm{rad}$.

The evolution function $\vec{S}_{3}(t)$ [Eq. (100)] uses the ideal ring with solenoid $S_{1}$ switched $\mathrm{ON}\left(\chi_{\mathrm{rot}}^{\mathrm{Sol} 2}=0\right)$ and $\mathrm{rf}$ Wien filter. The calculated resonance strength $\varepsilon_{\mathrm{EDM}}$ in the angular range $\phi_{\mathrm{rot}}^{\mathrm{WF}}=\left[-0.1^{\circ}, \ldots,+0.1^{\circ}\right]$ and $\chi_{\mathrm{rot}}^{\text {Sol } 1}=\left[-0.1^{\circ}, \ldots,+0.1^{\circ}\right]$, shows a clearly visible shift of the pattern away from the origin [Fig. 20(b)]. The shift amounts to about $0.18^{\circ}=\sin \left(\xi_{\mathrm{EDM}}=0.3 \mathrm{mrad}\right)$. The relative uncertainties of the points shown in Fig. 20, obtained from the fits, range from $\Delta \varepsilon^{\mathrm{EDM}} / \varepsilon^{\mathrm{EDM}}=2.0 \times$ $10^{-5}$ to $4.1 \times 10^{-2}$.

For the set of points $\left(\phi_{\mathrm{rot}}^{\mathrm{WF}}, \chi_{\mathrm{rot}}^{\mathrm{Sol} 1}\right)$ shown in Fig. 20, the initial spin tunes $\nu_{s}$, i.e., before the rf Wien filter is turned on, are shown in Fig. 21. The result indicates the familiar

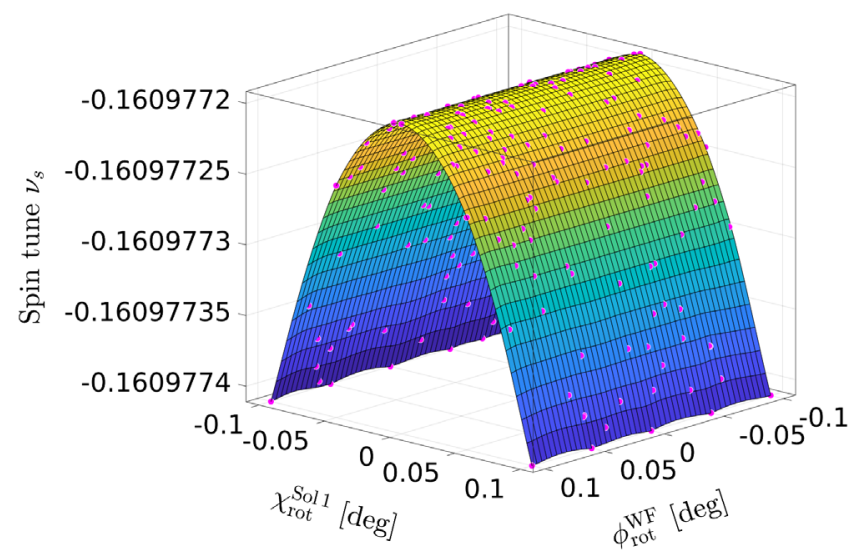

FIG. 21. Initial spin tunes $\nu_{s}$ for the angular intervals $\phi_{\mathrm{rot}}^{\mathrm{WF}}=$ $\chi_{\mathrm{rot}}^{\mathrm{Sol} 1}=\left[-0.1^{\circ}, \ldots,+0.1^{\circ}\right]$ for the data points $\left(\phi_{\mathrm{rot}}^{\mathrm{WF}}, \chi_{\mathrm{rot}}^{\mathrm{Sol} 1}\right)$ shown in Figs. 20(a) and 20(b) with an assumed EDM of $d=10^{-18} e \mathrm{~cm}$. quadratic dependence $\Delta \nu_{s}\left(\chi_{1}, \chi_{2}=0\right) \propto \chi_{1}^{2}$, as described by Eq. (103).

\section{G. Characterization of $\varepsilon^{\mathrm{EDM}}\left(\phi_{\text {rot }}^{\mathrm{WF}}, \chi_{\text {rot }}^{\text {Sol 1 }}\right)$}

\section{Operation of $r f$ Wien filter exactly on resonance}

In this section, the contour of the surface $\varepsilon^{\mathrm{EDM}}\left(\phi_{\mathrm{rot}}^{\mathrm{WF}}\right.$, $\left.\chi_{\text {rot }}^{\text {Sol } 1}\right)$, shown in Fig. 20(a), is compared to the theoretical expectation, given in Eq. (A5). The functional dependence describes a quadratic surface, also know as elliptic paraboloid, and is used here in the form of

$$
\begin{aligned}
\left(\varepsilon^{\mathrm{EDM}}\right)^{2}= & A \cdot\left(\phi_{\mathrm{rot}}^{\mathrm{WF}}-\phi_{0}\right)^{2} \\
& +B \cdot\left(\frac{\chi_{\mathrm{rot}}^{\mathrm{Sol} 1}}{2 \sin \pi \nu_{s}^{(2)}}+\chi_{0}\right)^{2}+C,
\end{aligned}
$$

where the unperturbed spin tune $\nu_{s}^{(2)}$ for the EDM of $d=10^{-18} e \mathrm{~cm}$, assumed in the simulation, is given by

$$
\begin{aligned}
\nu_{s}^{(2)} & =-0.160977192137641, \quad \text { and } \\
2 \sin \pi \nu_{s}^{(2)} & =-0.968883216683076 .
\end{aligned}
$$

It should be emphasized that the simulations shown in Fig. 20 reflect the situation when the rf Wien filter is operated exactly on resonance. During the corresponding EDM experiments in the ring, however, a certain spin-tune feedback is imperative to maintain the resonance condition for long periods of time, i.e., the spin-precession frequency in Eq. (35), using the measured spin tune [27]. To maintain phase and frequency lock when the rf Wien filter is actively operating, turns out to be much more tricky, and more sophisticated approaches, beyond those outlined in [23], are presently being pursued by the JEDI collaboration. 


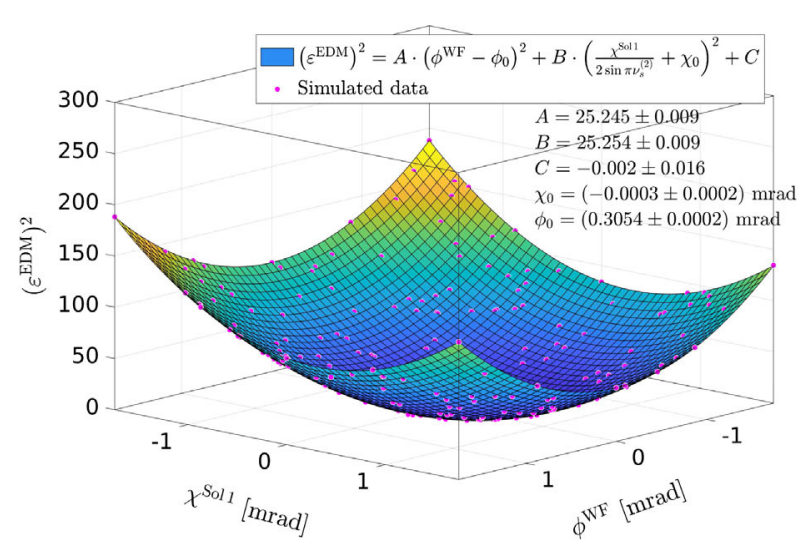

(a)

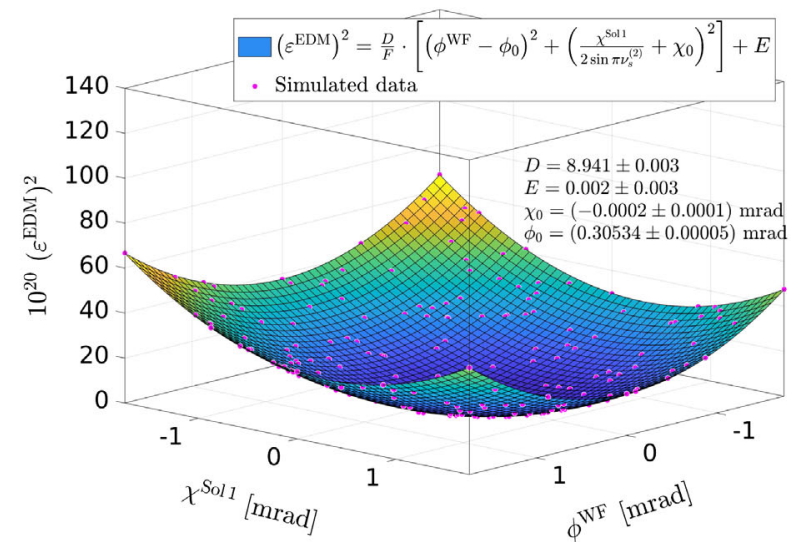

(b)

FIG. 22. Fits to the simulated data for the resonance strength $\left(\varepsilon^{\mathrm{EDM}}\right)^{2}$ as a function of $\left(\phi_{\mathrm{rot}}^{\mathrm{WF}}, \chi_{\mathrm{rot}}^{\mathrm{Sol} 1}\right)$.

Only such a phase and frequency lock during a measurement cycle enables one to take full advantage of the large spin-coherence time (SCT) of $\tau_{\mathrm{SCT}} \simeq 1000 \mathrm{~s}$, achieved by JEDI at COSY $[28,29]$.

The result of a fit without weighting is shown in Fig. 22(a). The fit parameters are listed in Table VII. It should be noted that within the uncertainties obtained from the fit, $A=B$, while $C$ and $\chi_{0}$ are compatible with zero. Here, $\chi_{0}$ represents a primordial tilt of the stable spin axis at the rf Wien filter along the horizontal axis, $c_{x}$. For the model ring, one would expect

$$
\chi_{0}=0=c_{x},
$$

a property which is nicely returned by the fit shown in Fig. 22(a).

In addition, the fit to the simulated data is expected to return $\phi_{0}=\left|\xi_{\mathrm{EDM}}\left(d=10^{-18} e \mathrm{~cm}\right)\right|=0.3054 \mathrm{mrad}$, given by Eq. (11), and the fitted result

$$
\phi_{0}=(0.3054 \pm 0.0002) \mathrm{mrad}
$$

returns this value accurately.

\section{Validation of the scale of $\varepsilon^{\mathrm{EDM}}$}

The fit with the elliptic paraboloid, shown in Fig. 22(a), indicates that the surface is described with $A=B$. In the

TABLE VII. Parameters of the fit shown in Fig. 22(b). The fit parametrizes the simulated data shown in Fig. 20(a), using Eq. (115) and $F=10^{20}$.

\begin{tabular}{lc}
\hline \hline Fit parameter & Value \\
\hline$A$ & $25.245 \pm 0.009$ \\
$B$ & $25.254 \pm 0.009$ \\
$C$ & $-0.002 \pm 0.0016$ \\
$\chi_{0}$ & $(0.0003 \pm 0.0002) \mathrm{mrad}$ \\
$\phi_{0}$ & $(0.3054 \pm 0.0002) \mathrm{mrad}$ \\
\hline \hline
\end{tabular}

following, the first fit function from Eq. (111) is slightly altered, yielding

$\left(\varepsilon^{\mathrm{EDM}}\right)^{2}=\frac{D}{F} \cdot\left[\left(\phi_{\mathrm{rot}}^{\mathrm{WF}}-\phi_{0}\right)^{2}+\left(\frac{\chi_{\mathrm{rot}}^{\mathrm{Sol} 1}}{2 \sin \pi \nu_{s}^{(2)}}+\chi_{0}\right)^{2}\right]+E$,

where a factor $F=10^{20}$ has been introduced to scale the resonance strength. The second fit now uses weights derived from the uncertainty of the fitted $\Omega^{p_{y}}$ using Eq. (106). The fit obtained is shown in Fig. 22(b), and the results are summarized in Table VIII. The agreement between the theoretical model and the simulated data is good, the $\chi^{2} / \mathrm{ndf}=374.4 / 194=1.9$.

According to Eq. (A5), the factor in front of the brackets in Eq. (115) reads

$$
\frac{D}{F}=k \stackrel{!}{=} \frac{\psi_{\mathrm{WF}}^{2}}{16 \pi^{2}},
$$

where the Wien filter rotation angle $\psi_{\mathrm{WF}}$ from Eq. (34) is used. Inserting the numerical value of $D$ from the fit (Table VIII), and taking into account that the results are in $\mathrm{mrad}$, the ratio

TABLE VIII. Fit parameters obtained using Eq. (115) on the simulation data shown in Fig. 22(b), using factor $F=10^{20}$.

\begin{tabular}{lc}
\hline \hline Fit parameter & Value \\
\hline$D$ & $8.941 \pm 0.003$ \\
$E$ & $0.002 \pm 0.003$ \\
$\chi_{0}$ & $(-0.0002 \pm 0.0001) \mathrm{mrad}$ \\
$\phi_{0}$ & $(0.30534 \pm 0.00005) \mathrm{mrad}$ \\
$\chi^{2} / \mathrm{ndf}$ & $1.9=374.4 / 194$ \\
\hline \hline
\end{tabular}




$$
\frac{D \cdot 10^{6}}{F \cdot k}=9.9954 \times 10^{-1}
$$

yields the expected value near unity, which validates the scaling factor in Eq. (A5).

The second fit yields a similar value for

$$
\begin{aligned}
\phi_{0} & =(0.30534 \pm 0.00005) \mathrm{mrad} \\
& \approx\left|\xi_{\mathrm{EDM}}\left(d=10^{-18} e \mathrm{~cm}\right)\right|,
\end{aligned}
$$

compared to the first one, shown in Fig. 22(a), and $\chi_{0}$ and $C$ are both compatible with zero.

\section{CONCLUSIONS AND OUTLOOK}

The $\mathbf{S O}(3)$ matrix formalism used here to describe the spin rotations on the closed orbit, i.e., the spin dynamics of the interplay of an $\mathrm{rf}$ Wien filter with a machine lattice that includes solenoids, proved very valuable. The general features of the deuteron EDM experiment at COSY can be obtained rather immediately.

The polarization evolution in the ring in the presence of an rf Wien filter that is operated on a parametric resonance, in terms of the resonance tune or resonance strength $\varepsilon^{\mathrm{EDM}}$ is theoretically well understood. This will allow us to investigate in the future effects of increasingly smaller magnetic imperfections, either through additional solenoidal fields in the ring, or by transverse magnetic fields via the rotation of the rf Wien filter around the beam axis.

In the near future, it is planned to incorporate into the developed matrix formalism also dipole magnet displacement and rotation parameters, available from a recent survey at COSY. This will allow us to determine the orientation of the stable spin axis of the machine at the location of the rf Wien filter, and to extract the EDM from a measurement of the resonance strengths as a function of $\left(\phi_{\mathrm{rot}}^{\mathrm{WF}}, \chi_{\mathrm{rot}}^{\mathrm{Sol} 1}\right)$. In addition, it shall be possible to incorporate the spin rotations from misplaced and rotated quadrupole magnets on the closed orbit into the formalism as well. Of course, the approach taken is no substitute for more advanced spin-tracking codes, but the results obtained here can be applied to benchmark those codes.

It should be noted that the JEDI collaboration is presently applying beam-based alignment techniques to improve the knowledge about the absolute beam positions in COSY [15]. Once the orbit corrections based on the results of the beam-based alignment have been implemented, the approach described here to parametrize the spin rotations solely on the basis of the closed orbit, will become even more realistic.

The collaboration devoted a considerable effort to the experimental optimization of the spin-coherence time $[28,29]$. For deuterons at momenta near $970 \mathrm{MeV} / c$, spin-coherence times in excess of $1000 \mathrm{~s}$ are routinely achieved nowadays by careful adjustment of the sextupole magnets in the ring. The collaboration is presently preparing the corresponding investigations for protons.

An obvious limitation of the analytic treatment presented here, is the implicit assumption that the beam emittance is vanishing and correspondingly, the spin-coherence time is infinitely large. In the near future, we intend to apply more sophisticated spin-tracking algorithms to better understand the relation between a finite beam emittance and the corresponding spin-coherence time, both for deuteron and proton beams. It remains to be seen, how far one can develop analytic descriptions to actually model the spin-coherence time and other spin dynamics aspects of beams with finite emittance.

A full-fledged analytic treatment of decoherence of the polarization in a beam bunch due to synchrotron oscillations was reported in [30], and these findings will be applied to the analysis of the experimental data. Yet another potential source of decoherence is intrabeam scattering, which evidently randomizes the synchrotron motion and thereby the spin phases of the stored particles. Effects from intrabeam scattering can be readily incorporated in the formalism exposed in [30].

Recently, in conjunction with the design of the rf Wien filter [5], an approach based on the polynomial chaos expansion has been successfully applied to determine a hierarchy of uncertainties. Such a methodology, in combination with the spin-tracking approach based on the matrix formalism outlined here, can be employed to efficiently generate a hierarchy of uncertainties for the EDM prototype ring [11], based on the design parameters of the machine.

The employed spin-tracking approach shall be also applied to study various aspects of the presently applied spin-tune feedback system, which is used to phase lock the spin vector to the rf of the Wien filter [23].

\section{ACKNOWLEDGMENTS}

This work has been performed in the framework of the JEDI collaboration and is supported by an ERC AdvancedGrant of the European Union (Proposal No. 694340). The work of N. N. N. was a part of the Russian Ministry of Science Program No. 0033-2019-0005. Numerous discussions with colleagues went into this document, foremost we would like to acknowledge those with Volker Hejny, Alexander Nass, Jörg Pretz, Artem Saleev, and Helmut Soltner. We would especially like to thank Volker Hejny for his very attentive reading of the manuscript.

\section{APPENDIX: DEPENDENCE OF THE EDM RESONANCE STRENGTH ON $\phi^{\mathrm{WF}}$ AND $\chi^{\mathrm{Sol} 1}$}

The functional dependence of a physical rotation of the Wien filter around the beam axis by $\phi_{\mathrm{rot}}^{\mathrm{WF}}$ and of a spin rotation in static solenoids (see Fig. 4) by $\chi_{\mathrm{rot}}^{\mathrm{Sol}_{1,2}}$ on the resonance strength $\varepsilon^{\mathrm{EDM}}$ [Eq. (106)] is discussed. 
At the location of the polarimeter, only the vertical and radial components of the beam polarization $\left[S_{y}(t)\right.$ and $S_{x}(t)$ ] can be determined. At the rf Wien filter, the orientation of the stable spin axis is denoted by $\vec{c}$, and in EDM mode the direction of the magnetic field by $\vec{n}_{\mathrm{WF}}$ [see Eq. (39)]. The in-plane $S_{x}(t)$ thus obviously depends on $\left[\vec{n}_{\mathrm{WF}} \times \vec{c}\right]$.

In an ideal all-magnetic ring under consideration, the stable spin axis is close to the vertical direction $\vec{e}_{y}$,

$$
\begin{aligned}
\vec{c} & =\cos \xi_{\mathrm{EDM}} \cdot \vec{e}_{y}+\sin \xi_{\mathrm{EDM}} \cdot \vec{e}_{x} \\
& \approx \vec{e}_{y}+\xi_{\mathrm{EDM}} \cdot \vec{e}_{x} .
\end{aligned}
$$

In EDM mode, the magnetic axis of the rf Wien filter can be approximated by

$$
\begin{aligned}
\vec{n}_{\mathrm{WF}} & =\cos \phi_{\mathrm{rot}}^{\mathrm{WF}} \cdot \vec{e}_{y}+\sin \phi_{\mathrm{rot}}^{\mathrm{WF}} \cdot \vec{e}_{x} \\
& \approx \vec{e}_{y}+\phi_{\mathrm{rot}}^{\mathrm{WF}} \cdot \vec{e}_{x} .
\end{aligned}
$$

The stable spin axis $\vec{c}$ can be manipulated by static solenoids in the ring, and the drift solenoids $S_{1}$ and $S_{2}$ of the electron coolers (or the Siberian snake instead of $S_{1}$ ) generate the spin kicks $\chi_{1}$ and $\chi_{2}$. When both solenoids $S_{1,2}$ are turned on, one can write for the stable spin axis

$$
\begin{aligned}
c_{x} & =\xi_{\mathrm{EDM}}+\frac{1}{2} \chi_{2}, \\
c_{z} & =\frac{1}{2 \sin \pi \nu_{s}}\left(\chi_{1}+\chi_{2} \cos \pi \nu_{s}\right) .
\end{aligned}
$$

In case solenoid $S_{2}$ is off $\left(\chi_{2}=0\right)$, one obtains

$$
\left[\vec{n}_{\mathrm{WF}} \times \vec{c}\right]=\left(\xi_{\mathrm{EDM}}-\phi_{\mathrm{rot}}^{\mathrm{WF}}\right) \vec{e}_{x}+\frac{\chi_{1}}{2 \sin \pi \nu_{s}} \vec{e}_{z}
$$

Thus the resonance strength squared can be written as a sum of two independent quadratic functions,

$\left(\epsilon^{\mathrm{EDM}}\right)^{2}=\frac{\psi_{\mathrm{WF}}^{2}}{16 \pi^{2}}\left[\left(\xi_{\mathrm{EDM}}-\phi_{\mathrm{rot}}^{\mathrm{WF}}\right)^{2}+\left(\frac{\chi_{1}}{2 \sin \pi \nu_{s}}\right)^{2}\right]$,

where $\psi_{\mathrm{WF}}$ is defined in Eq. (34).

[1] C. Weidemann et al., Toward polarized antiprotons: Machine development for spin-filtering experiments, Phys. Rev. ST Accel. Beams 18, 020101 (2015).

[2] JEDI Collaboration, proposals available from http:// collaborations.fz-juelich.de/ikp/jedi/.

[3] F. Rathmann and N. N. Nikolaev, Electric dipole moment searches using storage rings, Proc. Sci., SPIN2018 (2019) 004.

[4] J. Slim, R. Gebel, D. Heberling, F. Hinder, D. Hölscher, A. Lehrach, B. Lorentz, S. Mey, A. Nass, F. Rathmann, L.
Reifferscheidt, H. Soltner, H. Straatmann, F. Trinkel, and J. Wolterse, Electromagnetic simulation and design of a novel waveguide $\mathrm{rf}$ wien filter for electric dipole moment measurements of protons and deuterons, Nucl. Instrum. Methods Phys. Res., Sect. A 828, 116 (2016).

[5] J. Slim, F. Rathmann, A. Nass, H. Soltner, R. Gebel, J. Pretz, and D. Heberling, Polynomial Chaos Expansion method as a tool to evaluate and quantify field homogeneities of a novel waveguide rf Wien filter, Nucl. Instrum. Methods Phys. Res., Sect. A 859, 52 (2017).

[6] J. Slim, F. Rathmann, and D. Heberling, Computational framework for particle and spin simulations based on the stochastic Galerkin method, Phys. Rev. E96, 063301 (2017).

[7] F. Rathmann, A. Saleev, and N. N. Nikolaev, The search for electric dipole moments of light ions in storage rings, J. Phys. Conf. Ser. 447, 012011 (2013).

[8] W. M. Morse, Y. F. Orlov, and Y. K. Semertzidis, rf Wien filter in an electric dipole moment storage ring: The "partially frozen spin" effect, Phys. Rev. ST Accel. Beams 16, 114001 (2013).

[9] srEDM Collaboration, proposal available from http://www .bnl.gov/edm/files/pdf/proton_EDM_proposal_20111027_ final.pdf.

[10] V. Anastassopoulos et al., A storage ring experiment to detect a proton electric dipole moment, Rev. Sci. Instrum. 87, 115116 (2016).

[11] F. Abusaif et al., Storage ring to search for electric dipole moments of charged particles-Feasibility study (2019), https://arxiv.org/abs/1912.07881.

[12] S. Y. Lee, Spin Dynamics and Snakes in Synchrotrons (World Scientific, Singapore, 1997).

[13] S. R. Mane, Y. M. Shatunov, and K. Yokoya, Spin-polarized charged particle beams in high-energy accelerators, Rep. Prog. Phys. 68, 1997 (2005).

[14] N. N. Bogoliubov and Y. A. Mitropolsky, Asymptotic Methods in the Theory of Non-linear Oscillations, International Monographs on Advanced Mathematics and Physics (Gordon and Breach, New York, 1961).

[15] T. Wagner, Beam time request on beam-based alignment, available from http://collaborations.fz-juelich.de/ikp/jedi/ public_files/proposals/ Proposal_Beam_Based_Alignment_CBAC_10.pdf.

[16] T. Fukuyama and A. J. Silenko, Derivation of generalized Thomas-Bargmann-Michel-Telegdi equation for a particle with electric dipole moment, Int. J. Mod. Phys. A 28, 1350147 (2013).

[17] NIST database, available from http://physics.nist.gov/cuu/ Constants/index.html.

[18] A. Saleev et al. (JEDI Collaboration), Spin tune mapping as a novel tool to probe the spin dynamics in storage rings, Phys. Rev. Accel. Beams 20, 072801 (2017).

[19] E. Kovács, Rotation about an arbitrary axis and reflection through an arbitrary plane, Annales Societatis Mathematicae Polonae Series IV, Fundamenta informaticae 40, 175 (2012); http://ami.ektf.hu/uploads/papers/finalpdf/AMI_40_ from175to186.pdf

[20] L. Landau and E. Lifshitz, Mechanics, 1 (Elsevier Science, New York, 1982).

[21] T. Fossen and H. Nijmeijer, Parametric Resonance in Dynamical Systems (Springer, New York, 2011). 
[22] A. Jenkins, Self-oscillation, Phys. Rep. 525, 167 (2013).

[23] N. Hempelmann et al. (JEDI Collaboration), Phase Locking the Spin Precession in a Storage Ring, Phys. Rev. Lett. 119, 014801 (2017).

[24] N. Hempelmann et al. (JEDI Collaboration), Phase measurement for driven spin oscillations in a storage ring, Phys. Rev. Accel. Beams 21, 042002 (2018).

[25] J. M. Pendlebury et al., Revised experimental upper limit on the electric dipole moment of the neutron, Phys. Rev. D 92, 092003 (2015).

[26] S. Afach et al., Measurement of a false electric dipole moment signal from ${ }^{199} \mathrm{Hg}$ atoms exposed to an inhomogeneous magnetic field, Eur. Phys. J. D 69, 225 (2015).

[27] D. Eversmann et al. (JEDI Collaboration), New Method for a Continuous Determination of the Spin Tune in Storage
Rings and Implications for Precision Experiments, Phys. Rev. Lett. 115, 094801 (2015).

[28] G. Guidoboni et al. (JEDI Collaboration), How to Reach a Thousand-Second in-Plane Polarization Lifetime with $0.97-$ Gev/c Deuterons in a Storage Ring, Phys. Rev. Lett. 117, 054801 (2016).

[29] G. Guidoboni et al. (JEDI Collaboration), Connection between zero chromaticity and long in-plane polarization lifetime in a magnetic storage ring, Phys. Rev. Accel. Beams 21, 024201 (2018).

[30] A. Saleev, N. N. Nikolaev, F. Rathmann, F. Hinder, J. Pretz, and M. Rosenthal, , Pis'ma Zh. Eksp. Teor. Fiz. 106, 199 (2017) [Non-exponential decoherence of radiofrequency resonance rotation of spin in storage rings, JETP Lett. 106, 213 (2017)]. 